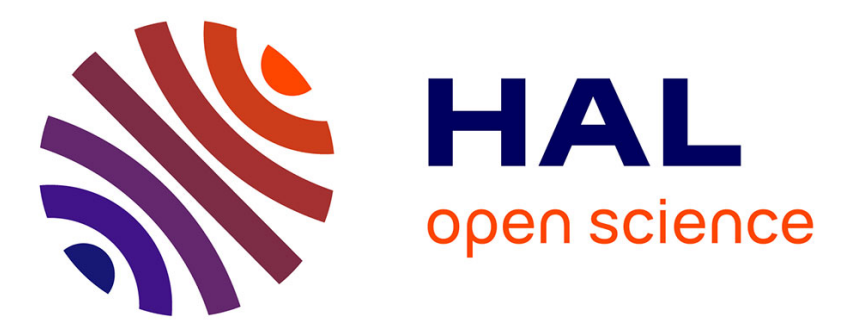

\title{
Integer programming formulations and efficient local search for relaxed correlation clustering
}

Eduardo Queiroga, Anand Subramanian, Rosa Figueiredo, Yuri Y. Frota

\section{To cite this version:}

Eduardo Queiroga, Anand Subramanian, Rosa Figueiredo, Yuri Y. Frota. Integer programming formulations and efficient local search for relaxed correlation clustering. Journal of Global Optimization, In press, 10.1007/s10898-020-00989-7 . hal-03141558

\author{
HAL Id: hal-03141558 \\ https://hal.science/hal-03141558
}

Submitted on 25 Feb 2021

HAL is a multi-disciplinary open access archive for the deposit and dissemination of scientific research documents, whether they are published or not. The documents may come from teaching and research institutions in France or abroad, or from public or private research centers.
L'archive ouverte pluridisciplinaire HAL, est destinée au dépôt et à la diffusion de documents scientifiques de niveau recherche, publiés ou non, émanant des établissements d'enseignement et de recherche français ou étrangers, des laboratoires publics ou privés. 


\title{
Integer programming formulations and efficient local search for relaxed correlation clustering
}

\author{
Eduardo Queiroga • Anand Subramanian . \\ Rosa Figueiredo · Yuri Frota
}

Received: date / Accepted: date

\begin{abstract}
Relaxed correlation clustering (RCC) is a vertex partitioning problem that aims at minimizing the so-called relaxed imbalance in signed graphs. RCC is considered to be an NP-hard unsupervised learning problem with applications in biology, economy, image recognition and social network analysis. In order to solve it, we propose two linear integer programming formulations and a local searchbased metaheuristic. The latter relies on auxiliary data structures to efficiently perform move evaluations during the search process. Extensive computational experiments on existing and newly proposed benchmark instances demonstrate the superior performance of the proposed approaches when compared to those available in the literature. While the exact approaches obtained optimal solutions for open problems, the proposed heuristic algorithm was capable of finding high quality solutions within a reasonable CPU time. In addition, we also report improving results for the symmetrical version of the problem. Moreover, we show the benefits of implementing the efficient move evaluation procedure that enables the proposed metaheuristic to be scalable, even for large-size instances.
\end{abstract}

Keywords Relaxed correlation clustering · unsupervised learning · integer programming $\cdot$ iterated local search

E. Queiroga and Y. Frota

Instituto de Computação, Universidade Federal Fluminense, Rua Passo da Pátria, São Domingos, 24210-240, Niterói-RJ, Brazil.

E-mail: eduardoqueiroga@id.uff.br, eduardovqueiroga@gmail.com, yuri@ic.uff.br Phone: +55 (21) 2629-5637

A. Subramanian

Departamento de Sistemas de Computação, Centro de Informática, Universidade Federal da Paraíba, Rua dos Escoteiros, Mangabeira, 58055-000, João Pessoa-PB, Brazil.

E-mail: anand@ci.ufpb.br

R. Figueiredo

Laboratoire Informatique d'Avignon, Avignon Université, 339 Chemin des Meinajaries, 84911, Avignon cedex 9, France.

E-mail: rosa.figueiredo@univ-avignon.fr 


\section{Introduction}

In graph theory, signed graphs are those where each arc (or edge) has a positive or negative sign $[50,51]$. This type of graph has been extensively used for modeling problems in various fields including biology [16], economy [29, 47], chemistry [38], ecology [15], image segmentation [31], linguistics [46], but mainly in social network analysis $[19,23,2,22,3,10]$. One of these problems is correlation clustering (CC) [5], which is a well-known unsupervised learning problem that aims at finding a vertex partitioning in a signed graph so as to minimize the disagreements, given by negative arcs (or edges) within a cluster and positive arcs (or edges) between clusters. Before formally defining the CC problem on a directed signed graph, we shall introduce some notation.

- Let $G=(V, A, s)$ be a signed digraph, where $V=\{1,2, \ldots, n\}$ is the vertex set, $A \subseteq V \times V$ is the arc set, and $s: A \rightarrow\{+,-\}$ is a function that assigns a sign to each arc.

- An arc $a \in A$ is called negative if $s(a)=-$ and positive if $s(a)=+$.

- For each arc $a \in A$, let $w_{a}$ be an associated non-negative weight. We will also use $w_{i j}$ and $w_{j i}$ to denote the weight of the $\operatorname{arcs}(i, j)$ and $(j, i)$, respectively.

- The set of positive and negative arcs are denoted, respectively, as $A^{+}$and $A^{-}$; thus $A=A^{-} \cup A^{+}$.

- A partition of $V$ into $l$ disjoint subsets $P=\left\{S_{1}, S_{2}, \ldots, S_{l}\right\}$ is called a $l$ partition of $V$.

- For $1 \leq p, q \leq l$, let $A\left[S_{p}: S_{q}\right]=\left\{(i, j) \in A \mid i \in S_{p}, j \in S_{q}\right\}$.

- $\Omega^{+}\left(S_{p}, S_{q}\right)=\sum_{a \in A^{+} \cap A\left[S_{p}: S_{q}\right]} w_{a}$ and $\Omega^{-}\left(S_{p}, S_{q}\right)=\sum_{a \in A^{-} \cap A\left[S_{p}: S_{q}\right]} w_{a}$.

The imbalance $I(P)$ of a $l$-partition $P$ is defined as

$$
I(P)=\sum_{1 \leq p \leq l} \Omega^{-}\left(S_{p}, S_{p}\right)+\sum_{\substack{1 \leq p \leq l, 1 \leq q \leq l, p \neq q}} \Omega^{+}\left(S_{p}, S_{q}\right)
$$

The CC problem consists of determining a partition $P$ which minimizes $I(P)$. One of the applications of $\mathrm{CC}$ is related to the analysis of structural balance on social networks. In such networks, the arcs represent social relations between actors (i.e. the vertices of the network), whereas the sign represents feelings such as like/dislike and agreement/disagreement. According to the structural balance theory of Heider [30, 14], a network is balanced if there is a bipartition of the vertex set so that every positive arc joins actors in a same group and every negative arc joins actors in different groups. Later, Davis [17] generalized the structural balance to support a partition with more than two groups, which is fully compatible with the criterion optimized by the CC. Indeed, an algorithm for CC is a useful tool for evaluating how balanced a social network is. Note that a signed graph is balanced if there is a partition $P$ such that $I(P)=0$.

Although traditional structural balance works in several scenarios, Doreian and Mrvar [20] pointed out that this concept could not be appropriate for some networks. They argued that Equation (1) penalizes patterns in the partitions associated with relevant social psychological processes. For example, the network in Figure 1a represents a scenario with three groups, where one of them is a group of mutually hostile mediators (vertices 8,9 , and 10). Figure $1 \mathrm{~b}$ illustrates why CC is 


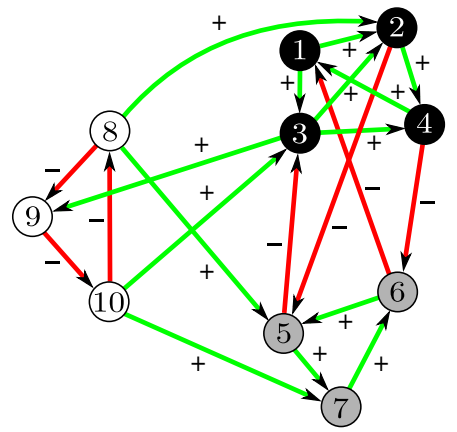

(a) Ideal partition

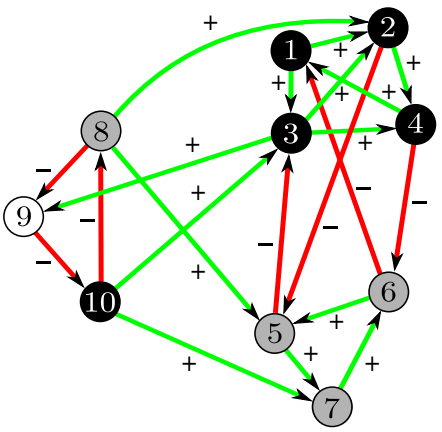

(b) CC partition

Fig. 1: Structural balance in a network with mutually hostile mediators.

not suitable in this case: it is not capable of detecting the group of mediators or any type of subgroup internal hostility. This was illustrated in practice by Levorato et al. [36], where positive/negative mediation was detected in networks describing the United Nations General Assembly Voting Data. The equivalent was also verified for the case of differential popularity (a process in which some actors receive more positive links than others in a group) detected in benchmark instances from the literature [21], and for internal hostility detected in networks describing voting activity of members of the European Parliament [3].

Still in [20], Doreian and Mrvar introduced the concept of generalized structural balance giving rise to a new definition for the imbalance of a vertex partition which corrects the partition patterns penalized in Equation (1). The relaxed imbalance of a $l$-partition partition $P$, denoted here $R I(P)$, is defined as

$$
R I(P)=\sum_{1 \leq p \leq l} \min \left\{\Omega^{+}\left(S_{p}, S_{p}\right), \Omega^{-}\left(S_{p}, S_{p}\right)\right\}+\sum_{\substack{1 \leq p \leq l, 1 \leq q \leq l, p \neq q}} \min \left\{\Omega^{+}\left(S_{p}, S_{q}\right), \Omega^{-}\left(S_{p}, S_{q}\right)\right\}
$$

The main subject of this work is the relaxed correlation clustering (RCC) problem $[20,26]$, which is a CC variant. In the RCC, given an integer parameter $1 \leq k \leq n$, one aims at finding a partition $P \in \cup_{l=1}^{k} \mathcal{P}^{l}$ that minimizes the relaxed imbalance given by Equation (2), such that $\mathcal{P}^{l}$ is the set of all $l$-partitions of $V$. The optimal value of the RCC problem determines how balanced a network is w.r.t. the relaxed structural balance introduced by Doreian and Mrvar[20]. For example, the partition in Figure 1a is an optimal solution for RCC because $R I(P)=0$. Both $\mathrm{CC}$ and RCC problems were proven NP-hard by Bansal et al. [5] and Figueiredo and Moura [26], respectively.

Doreian and Mrvar [20] tackled the RCC by applying a relocation algorithm to analyze four real data sets related to relaxed structural balance, with up to 20 vertices. The authors adapted a heuristic method proposed in Doreian and Mrvar [19] for the CC problem with a fixed number of clusters, which optimizes a generic and parameterized function called criterion function. Later, Brusco et al. [9] proposed a branch-and-bound algorithm for solving RCC to optimality. This algorithm was capable of solving instances with up to 29 vertices (hereafter referred 
to as small-sized instances) and $k$ varying from 2 to 7 . Moreover, an additional set of instances with up to 40 vertices was considered for experiments with $k=\{3,5\}$. Figueiredo and Moura [26] developed an integer linear programming (ILP) formulation that was capable of solving some small-sized instances when $k=\{2,3\}$ and for high values of $k$, concluding that the proposed ILP formulation and the existing branch-and-bound algorithm are somewhat complementary approaches. The authors also proposed a symmetrical version of RCC (SRCC). Although different heuristic procedures were proposed in the literature for CC (see [19, 49, 48, 6, 36], among others), to the best of our knowledge, only one heuristic procedure has been applied to the RCC. Levorato et al. [36] adapted their iterated local search (ILS) algorithm, originally developed for CC, to solve SRCC. Thus, there are no heuristic or metaheuristic procedures specifically proposed for RCC problem.

The two main contributions can be summarized as follows:

- We present two novel integer programming formulations for the RCC problem and we investigate their empirical performance in comparison to an existing formulation. The results show that the new formulations appear to produce better results in practice.

- We propose a local search-based metaheuristic that relies on a series of auxiliary data structures to efficiently recalculate the relaxed imbalance after applying an operation that modifies a partition, as well as on a novel perturbation mechanism. The results obtained suggest that the developed algorithm is superior to an existing approach, producing, on average, high quality solutions in a limited amount of CPU time, not only for RCC instances but also for SRCC instances. Finally, we also demonstrate the practical benefits of implementing the move evaluation in an efficient way.

The remainder of the paper is organized as follows. Section 2 presents a small instance for the RCC problem. Section 3 defines the symmetric version of the problem. Section 4 introduces two novel mathematical formulations for the RCC. Section 5 explains the proposed efficient local search-based metaheuristic including the efficient move evaluation schemes. Section 6 presents the results of the computational experiments. Finally, the conclusions are discussed in Section 7.

\section{A small RCC example}

A small RCC example involving 6 vertices is depicted in Figure 2. In Figure 2(a), the signed graph to be partitioned is illustrated. In Figure 2(b), a feasible solution $P=\left\{S_{1}=\{1,2\}, S_{2}=\{3,4\}, S_{3}=\{5,6\}\right\}$ is presented. This solution has relaxed imbalance $R I(P)=4$ obtained by adding the following terms.

- $\min \left\{\Omega^{+}\left(S_{1}, S_{1}\right), \Omega^{-}\left(S_{1}, S_{1}\right)\right\}=\min \left\{\emptyset, w_{12}\right\}=\min \{0,1\}=0$

- $\min \left\{\Omega^{+}\left(S_{2}, S_{2}\right), \Omega^{-}\left(S_{2}, S_{2}\right)\right\}=\min \left\{w_{34}, \emptyset\right\}=\min \{1,0\}=0$

- $\min \left\{\Omega^{+}\left(S_{3}, S_{3}\right), \Omega^{-}\left(S_{3}, S_{3}\right)\right\}=\min \left\{w_{65}, w_{56}\right\}=\min \{1,1\}=1$

- $\min \left\{\Omega^{+}\left(S_{1}, S_{2}\right), \Omega^{-}\left(S_{1}, S_{2}\right)\right\}=\min \left\{w_{14}, w_{23}\right\}=\min \{1,1\}=1$

- $\min \left\{\Omega^{+}\left(S_{2}, S_{1}\right), \Omega^{-}\left(S_{2}, S_{1}\right)\right\}=\min \{\emptyset, \emptyset\}=\min \{0,0\}=0$

- $\min \left\{\Omega^{+}\left(S_{1}, S_{3}\right), \Omega^{-}\left(S_{1}, S_{3}\right)\right\}=\min \{\emptyset, \emptyset\}=\min \{0,0\}=0$

- $\min \left\{\Omega^{+}\left(S_{3}, S_{1}\right), \Omega^{-}\left(S_{3}, S_{1}\right)\right\}=\min \left\{w_{52}+w_{62}, w_{61}\right\}=\min \{2,1\}=1$

- $\min \left\{\Omega^{+}\left(S_{2}, S_{3}\right), \Omega^{-}\left(S_{2}, S_{3}\right)\right\}=\min \{\emptyset, \emptyset\}=\min \{0,0\}=0$ 


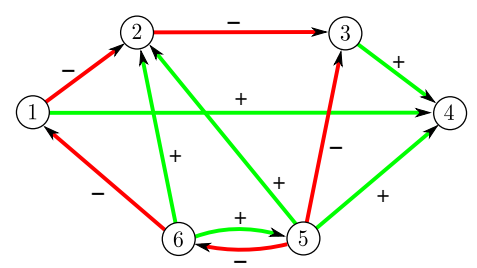

(a)

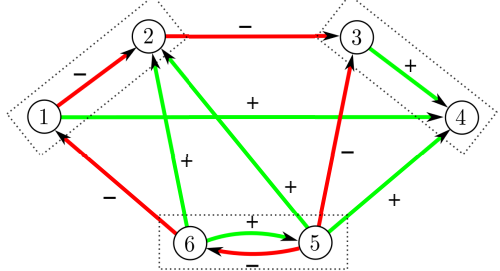

(b)

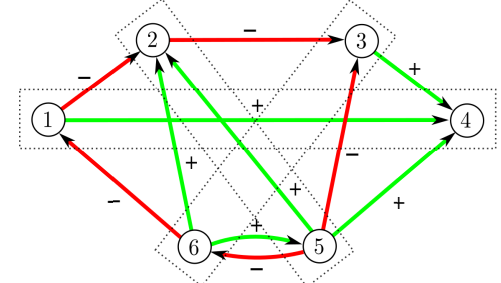

(c)

Fig. 2: (a) A small RCC instance with unitary weights and $k=3$. (b) A feasible solution $P=\{\{1,2\},\{3,4\},\{5,6\}\}$ with relaxed imbalance $R I(P)=4$. (c) An optimal solution $P^{*}=\{\{1,4\},\{2,5\},\{3,6\}\}$ with relaxed imbalance $R I(P)=1$.

- $\min \left\{\Omega^{+}\left(S_{3}, S_{2}\right), \Omega^{-}\left(S_{3}, S_{2}\right)\right\}=\min \left\{w_{53}, w_{54}\right\}=\min \{1,1\}=1$

Figure 2(c) depicts an optimal solution $P^{*}=\left\{S_{1}=\{1,4\}, S_{2}=\{2,5\}, S_{3}=\right.$ $\{3,6\}\}$ for the problem with $R I\left(P^{*}\right)=\min \left\{\Omega^{+}\left(S_{3}, S_{1}\right), \Omega^{-}\left(S_{3}, S_{1}\right)\right\}=$ $\min \left\{w_{34}, w_{61}\right\}=\min \{1,1\}=1$.

\section{Symmetric RCC}

In this work, we also consider the symmetric version of RCC (SRCC) introduced in Figueiredo and Moura [26]. The relaxed imbalance, as given by Equation (2), penalizes non-predominant relations (w.r.t. the signs) inside each cluster $q$ and non-predominant relations from a cluster $p$ to a cluster $q$. The difference in the symmetric relaxed imbalance defined in Figueiredo and Moura [26] is that it penalizes non-predominant relations among pairs of clusters, i.e., it considers simultaneously all positive (all negative) relations from $S_{p}$ to $S_{q}$ and from $S_{q}$ to $S_{p}$. Thus, the SRCC can be defined on an undirected graph in which parallel edges with opposite signs are allowed.

Let $G^{\prime}=\left(V, E, s^{\prime}\right)$ be an undirected signed graph with a positive weight $w_{i j}^{\prime}$ associated to each edge $\{i, j\} \in E$. Let us denote $E^{+}$and $E^{-}$, respectively, the sets of positive and negative edges in $E$; thus $E=E^{+} \cup E^{-}$. In this work, we transform the SRCC instance defined on $G^{\prime}=\left(V, E, s^{\prime}\right)$ into a RCC instance defined on a directed signed graph $G_{d}=(V, A, s)$ in which: $A=\{(i, j),(j, i):\{i, j\} \in E\}$; for each $(i, j) \in A, s((i, j))=s((j, i))=s^{\prime}(\{i, j\})$ and the associated weight $w_{i j}=w_{j i}=\frac{w_{i j}^{\prime}}{2}$. In other words, for each edge $\{i, j\} \in E$, one creates two arcs $(i, j),(j, i) \in A$ with the same signal and half of the weight. 
Let $E\left[S_{p}: S_{q}\right]$ be the set of edges connecting the vertices in $S_{p}$ and those in $S_{q}$. We denote $\Omega^{\prime+}\left(S_{p}, S_{q}\right)=\sum_{e \in E+\cap E\left[S_{p}: S_{q}\right]} w_{e}^{\prime}$ and $\Omega^{\prime-}\left(S_{p}, S_{q}\right)=$ $\sum_{e \in E^{-} \cap E\left[S_{p}: S_{q}\right]} w_{e}^{\prime}$. The symmetric relaxed imbalance $S R I(P)$ of a $l$-partition $P$ is defined as

$$
S R I(P)=\sum_{1 \leq p \leq l} \min \left\{\Omega^{\prime+}\left(S_{p}, S_{p}\right), \Omega^{\prime-}\left(S_{p}, S_{p}\right)\right\}+\sum_{1 \leq p<q \leq l} \min \left\{\Omega^{\prime+}\left(S_{p}, S_{q}\right), \Omega^{\prime-}\left(S_{p}, S_{q}\right)\right\}
$$

The following result relates Equations (2) and (3).

Proposition 1. Consider an undirected signed graph $G^{\prime}$ and the directed signed graph $G_{d}$ described above. Given any partitioning $P$, then $S R I(P)=R I(P)$.

Proof. First, we will show the equivalence of the first terms in (2) and (3), i.e. the intracluster imbalance. Then we will do the same for the second terms, i.e. for the intercluster imbalance. Let $S_{p}$ be any cluster in $P$. We have $\Omega^{\prime+}\left(S_{p}, S_{p}\right)=$ $\Omega^{+}\left(S_{p}, S_{p}\right)$ since, for each $e=\{i, j\} \in E^{+} \cap E\left[S_{p}: S_{p}\right],(i, j),(j, i) \in A^{+} \cap A\left[S_{p}\right.$ : $\left.S_{p}\right]$ with $w_{i j}^{\prime}=w_{i j}+w_{j i}$. The same can be argued for $\Omega^{\prime-}\left(S_{p}, S_{p}\right)$ and these two facts imply the equivalence of the first terms in (2) and (3). Now, let $S_{p}$ and $S_{q}$ be two clusters in $P$. By the definition of the directed graph $G_{d}$, we have $e=\{i, j\} \in E^{+} \cap E\left[S_{p}: S_{q}\right]$ iff $(i, j) \in A^{+} \cap A\left[S_{p}: S_{q}\right]$ and $(j, i) \in$ $A^{+} \cap A\left[S_{q}: S_{p}\right]$. Since, for each $e=\{i, j\} \in E, w_{i j}^{\prime}=w_{i j}+w_{j i}$, we have that $\Omega^{+}\left(S_{p}, S_{q}\right)=\Omega^{+}\left(S_{q}, S_{p}\right)=\Omega^{\prime+}\left(S_{p}, S_{q}\right) / 2$. The same holds for $\Omega^{-}\left(S_{p}, S_{q}\right)$ and, since the second term in (3) is written only for $p<q$, the equivalence of the second terms in (2) and (3) follows.

As a consequence of Proposition 1, solving the RCC over graph $G_{d}$ is equivalent to solving SRCC over $G^{\prime}$.

\section{Mathematical formulations}

Integer linear programming (ILP) problem formulations have been used to solve $\mathrm{CC}$ and other related problems defined on signed graphs [13, 12, 4, 28, 27]. For RCC, an ILP formulation was presented in [26]. When modeling vertex-clustering problems, if there is a need for keeping track the clusters used, two types of formulations can be adopted: cluster-indexed formulation $[24,7,11]$ or representatives formulation [1, 4, 13, 28]. Indeed, the ILP formulation of Figueiredo and Moura [26] is a representatives one. Next, we introduce two new formulations for RCC, one of each type.

4.1 Formulation F1: a cluster-indexed formulation

Let $K=\{1, \ldots, k\}$ be the set of possible cluster indexes. For each vertex $i \in V$ and $p \in K$ we define,

$x_{i}^{p}=\left\{\begin{array}{l}1, \text { if vertex } i \text { belongs to cluster } S_{p}, \\ 0, \text { otherwise. }\end{array}\right.$ 
A set of binary variables is used to describe the set of arcs that will be penalized once, according to Equation (2), the predominant relations are defined. For each $\operatorname{arc}(i, j) \in A$, we define,

$t_{i j}=\left\{\begin{array}{l}1, \text { if } \operatorname{arc}(i, j) \text { is penalized } \\ 0, \text { otherwise. }\end{array}\right.$

A set of binary variables is used to select if the imbalance from cluster $S_{p}$ to cluster $S_{q}$, with $p, q \in K$, is given by negative arcs (predominant relations are positive) or positive arcs (predominant relations are negative). Notice that intracluster imbalance is defined whenever $p=q$. For each pair of cluster indexes $p, q \in K$, we define,

$s_{p q}=\left\{\begin{array}{l}1, \text { if positive arcs from cluster } S_{p} \text { to cluster } S_{q} \text { are penalized, } \\ 0, \text { if negative arcs from cluster } S_{p} \text { to cluster } S_{q} \text { are penalized. }\end{array}\right.$

The formulation can be written as

$$
\begin{array}{lr}
\operatorname{minimize} & \sum_{(i, j) \in A} w_{i j} t_{i j} \\
\text { s.t: } \sum_{p \in K} x_{i}^{p}=1, & \forall i \in V, \\
t_{i j} \geq x_{i}^{p}+x_{j}^{q}-2+s_{p q}, & \forall(i, j) \in A^{+}, \forall p, q \in K, \\
t_{i j} \geq x_{i}^{p}+x_{j}^{q}-2+\left(1-s_{p q}\right), & \forall(i, j) \in A^{-}, \forall p, q \in K, \\
x_{i}^{p} \in\{0,1\}, & \forall i \in V, \forall p \in K, \\
t_{i j} \in\{0,1\}, & \forall(i, j) \in A, \\
s_{p q} \in\{0,1\}, & \forall p, q \in K .
\end{array}
$$

Objective function (4) minimizes the total relaxed imbalance. Constraints (5) ensure that each vertex is assigned exactly to one cluster. Constraints (6) and (7) define, respectively, if a positive or negative arc will be penalized due to assignment variables $x_{i}^{p}, x_{j}^{q}$ and the penalizing variables $s_{p q}$. Note that, when $p=q$, variable $t_{p q}$ defines an intracluster penalty. Finaly, constraints (8)-(10) define the domain of all variables.

Formulations that make use of cluster-indexed variables such as F1 are considered to be symmetric, as there are a substantial number of ways to represent the same partitioning with a different permutation of indices. In view of this, we add the following set of symmetry breaking inequalities introduced in Bulhões et al. [11] for the $p$-cluster editing problem: 


$$
\sum_{l=1}^{p} x_{i}^{l} \geq\left(\sum_{\substack{j \in V \\ j<i}} \sum_{l=1}^{p-1} x_{j}^{l}\right)-(i-2), \forall i \in V, \forall p \in K .
$$

The inequality above forbids the cluster containing $i$ to use a label superior to $p$ whenever each vertex $j<i$ is assigned to a cluster of index strictly smaller than $p$. The formulation in the next section adopts a similar strategy in order to break symmetry in the solution space.

\subsection{Formulation F2: a representatives formulation}

A representatives formulation for the RCC is presented as follows. The idea behind this kind of formulation [13] is the unique representation of a cluster by its vertex with the lowest label. Hence, for each pair of vertices $i, j \in V$ satisfying $i \leq j$, we define,

$x_{j}^{i}=\left\{\begin{array}{l}1, \text { if the vertex } j \text { is represented by vertex } i, \\ 0, \text { otherwise. }\end{array}\right.$

Note that when $i=j$, variable $x_{i}^{i}$ indicates if $i$ is a representative vertex.

Variables $s_{i j}$, with $i, j \in V$, used in F2 are equivalent to variables $s_{p q}$, with $p, q \in K$, used in F1. However now, vertices $i$ and $j$ are used to identify two clusters $(i \neq j)$ or one cluster $(i=j)$. Variables $t_{i j}$, with $(i, j) \in A$, are exactly the same as defined in F1. Hence, formulation F2 can be expressed as follows.

$$
\begin{array}{lr}
\operatorname{minimize} & \sum_{(i, j) \in A} w_{i j} t_{i j} \\
\text { s.t: } \sum_{i \in V: i \leq j} x_{j}^{i}=1, & \forall j \in V, \\
& x_{j}^{i} \leq x_{i}^{i}, \\
\sum_{i \in V} x_{i}^{i} \leq k, & \forall i, j \in V, i<j, \\
t_{i j} \geq x_{i}^{u}+x_{j}^{v}-2+s_{u v}, & \\
& \forall(i, j) \in A^{+}, \forall u, v \in V, \\
& u \leq i, v \leq j, \\
t_{i j} \geq x_{i}^{u}+x_{j}^{v}-2+\left(1-s_{u v}\right), & \forall(i, j) \in A^{-}, \forall u, v \in V, \\
& u \leq i, v \leq j, \\
x_{j}^{i} \in\{0,1\}, & \forall i, j \in V, i \leq j, \\
t_{i j} \in\{0,1\}, & \forall(i, j) \in A, \\
s_{i j} \in\{0,1\}, & \forall i, j \in V .
\end{array}
$$


Constraints (12) impose that each vertex must be represented by exactly one vertex: either by itself or by another one with a smaller index. Constraints (13) enforce vertex $i$ to be a representative one whenever a vertex $j$ is represented by $i$. Constraint (14) imposes $k$ as an upper bound on the number of representative vertices, i.e., on the number of clusters in the partition. Constraints (15) and (16) are, respectively, equivalent to constraints (6) and (7) of formulation F1. Finally, constraints (17)-(19) are the binary constraints.

The number of variables and constraints of formulations F1 and F2 are illustrated in Table 1. Note that since the number of vertices of a graph is usually much greater than the number of clusters, formulation F1 is more compact than F2. On the other hand, formulation F2 succeeds in eliminating cluster indices from the representation which breaks symmetry from formulation [13].

Table 1: Number of variables and constraints of formulations F1 and F2

\begin{tabular}{l|c|c}
\hline & \#Variables & \#Constraints \\
\hline F1 & $\mathcal{O}\left(n k+|A|+k^{2}\right)$ & $\mathcal{O}\left(n+k^{2}\left|A^{+}\right|+k^{2}\left|A^{-}\right|\right)$ \\
F2 & $\mathcal{O}\left(2 n^{2}+|A|\right)$ & $\mathcal{O}\left(n+n^{2}+n^{2}\left|A^{+}\right|+n^{2}\left|A^{-}\right|\right)$ \\
\hline
\end{tabular}

\section{Proposed local search-based metaheuristic}

In this section, we propose a metaheuristic algorithm for the RCC based on the iterated local search (ILS) method [37]. The key concept of ILS is to combine local search strategies and perturbation mechanisms to escape from local optima. The metaheuristic introduced in Levorato et al. [36] for CC and adapted for the solution of SRCC instances is also an ILS method. Different from Levorato et al. [36], in this work, we propose the use of several complex neighborhoods and the use of advanced data structures which make the search process more efficient.

Algorithm 1 presents a general framework of a multi-start ILS, hereafter referred to as $\mathrm{ILS}_{\mathrm{RCC}}$, which has the following input parameters:

a) $I_{R}$ is the number of restarts of the metaheuristic;

b) $I_{I L S}$ is the maximum number of ILS iterations without improvements;

c) $I_{P}$ is the maximum number of moves performed by a perturbation mechanism.

For each restart, an initial solution is randomly generated (line 5) and such solution is possibly improved by alternately applying local search (line 9) and perturbation (line 13) strategies until the maximum number of iterations $\left(I_{I L S}\right)$ without improvement is achieved. Finally, the best solution found among all restarts is returned (line 18).

The local search procedure is based on variable neighborhood descent (VND) [40], which is a technique that systematically explores a sequence of neighborhood structures (see Section 5.2), searching for better solutions. A neighborhood structure (or simply neighborhood) defines a set of neighbor solutions from a current solution by applying a so-called move. When VND finds an improving move using a particular neighborhood, the solution is updated and the procedure restarts 


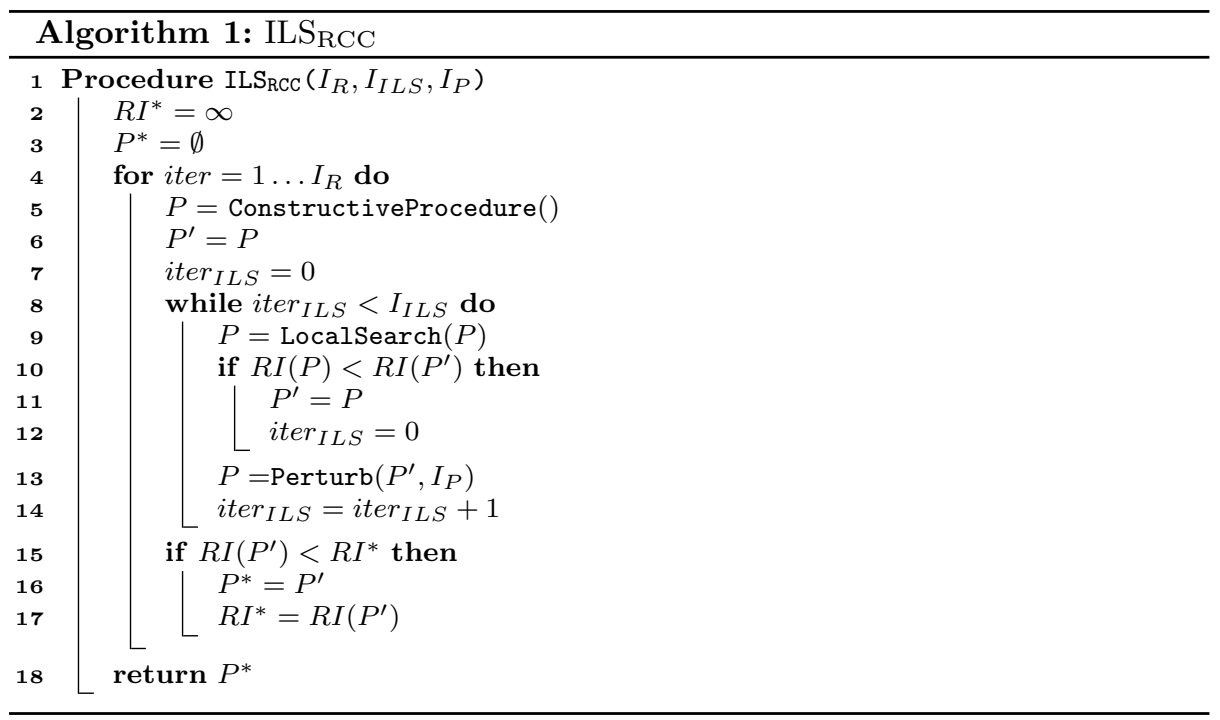

from the improved solution. The procedure terminates when all neighborhoods fail to improve the current solution. The best improvement strategy was adopted, i.e., a neighborhood is fully enumerated and the best improving move (if there is any) is applied. In addition, the neighborhood ordering is defined in a random fashion, which results in a strategy known as Randomized VND (RVND). The combination of ILS and RVND led to state-of-the-art methods for several important combinatorial optimization problems, such as: split-delivery vehicle routing problem [44], minimum latency problem [43] and minimizing weighted tardiness in single machine scheduling with sequence-dependent setup times [45].

The perturbation procedure randomly chooses one of the implemented mechanisms (see Section 5.3) in order to modify the local optimal solution $P^{\prime}$. The selected mechanism then applies $I_{P}$ random consecutive moves over $P^{\prime}$ in order to generate a solution to continue the search.

In what follows, we provide a detailed description of the auxiliary data structures used for performing efficient move evaluation, as well as on the neighborhood structures and perturbations mechanisms.

\subsection{Auxiliary data structures}

Assuming that an adjacency matrix is used to access the signed digraph $G$, and a feasible solution is represented by a set of subsets of indices (e.g. $P=\left\{S_{1}, S_{2}, S_{3}\right\}$, such that $S_{1}=\{1,2\}, S_{2}=\{3,4\}, S_{3}=\{5,6\}$ for a graph with 6 vertices; see Figure 2), the value of its associated objective function can be straightforwardly computed in $\mathcal{O}\left(l^{2} n^{2}\right)$ operations, where $l=|P|$. Note that this is due to the complexity of determining the intercluster imbalance. Consequently, performing the move evaluation of a neighbor solution from scratch every time during the local search may turn out to be computationally expensive, especially for large size 
instances. However, this can be done in a more efficient manner by precomputing and storing information in auxiliary data structures (ADSs).

We thus propose to implement two classes of ADSs: SumIntra[ $\left[S_{p}\right]$, which is a set of ADSs that stores the sum of the weights for different subsets of $A\left[S_{p}\right]$ (a.k.a. intracluster arcs of $S_{p}$ ); and SumInter $\left[S_{p}\right]\left[S_{q}\right]$, which is a set of ADSs that stores the sum of the weights for different subsets of $A\left[S_{p}: S_{q}\right]$ (a.k.a. known as intercluster arcs from $S_{p}$ to $S_{q}$ ). The ADSs are divided according to the sign and arc direction as described in Table 2 .

Table 2: Description of the proposed ADSs

\begin{tabular}{|c|c|}
\hline $\mathrm{ADS}$ & Description \\
\hline SumIntra $^{+}\left[S_{p}\right]=\sum_{a \in A}+\cap A\left[S_{p}\right] w_{a}$ & Sum of positive weights within $S_{p}$ \\
\hline SumIntra ${ }^{-}\left[S_{p}\right]=\sum_{a \in A^{-} \cap A\left[S_{p}\right]} w_{a}$ & Sum of negative weights within $S_{p}$ \\
\hline 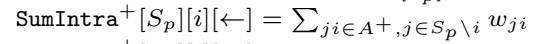 & Sum of positive weights from $S_{p} \backslash i$ to $i \in S_{p}$ \\
\hline 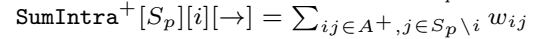 & Sum of positive weights from $i \in S_{p}$ to $S_{p} \backslash i$ \\
\hline SumIntra $^{-}\left[S_{p}\right][i][\leftarrow]=\sum_{j i \in A^{-}, j \in S_{p} \backslash i} w_{j i}$ & Sum of negative weights from $S_{p} \backslash i$ to $i \in S_{p}$ \\
\hline SumIntra ${ }^{-}\left[S_{p}\right][i][\rightarrow]=\sum_{i j \in A^{-}, j \in S_{p} \backslash i} w_{i j}$ & Sum of negative weights from $i \in S_{p}$ to $S_{p} \backslash i$ \\
\hline SumInter ${ }^{+}\left[S_{p}\right]\left[S_{q}\right]=\sum_{a \in A}+\cap A\left[S_{p}: S_{q}\right] w_{a}$ & Sum of positive weights from $S_{p}$ to $S_{q}$ \\
\hline SumInter $^{-}\left[S_{p}\right]\left[S_{q}\right]=\sum_{a \in A^{-} \cap A\left[S_{p}: S_{q}\right]} w_{a}$ & Sum of negative weights from $S_{p}$ to $S_{q}$ \\
\hline SumInter ${ }^{+}\left[S_{p}\right][i]\left[S_{q}\right][\rightarrow]=\sum_{i j \in A^{+}, j \in S_{q}} w_{i j}$ & Sum of positive weights from $i \in S_{p}$ to $S_{q}$ \\
\hline SumInter ${ }^{+}\left[S_{p}\right][i]\left[S_{q}\right][\leftarrow]=\sum_{j i \in A^{+}, j \in S_{q}} w_{j i}$ & Sum of positive weights from $S_{q}$ to $i \in S_{p}$ \\
\hline SumInter $\left[S_{p}\right][i]\left[S_{q}\right][\rightarrow]=\sum_{i j \in A^{-}, j \in S_{q}} w_{i j}$ & Sum of negative weights from $i \in S_{p}$ to $S_{q}$ \\
\hline SumInter ${ }^{-}\left[S_{p}\right][i]\left[S_{q}\right][\leftarrow]=\sum_{j i \in A^{-}, j \in S_{q}} w_{j i}$ & Sum of negative weights from $S_{q}$ to $i \in S_{p}$ \\
\hline
\end{tabular}

Given a feasible solution, the SumIntra and SumInter ADSs can be initially built in $\mathcal{O}\left(l^{2} n^{2}\right)$ operations as described in Algorithm 2.

\subsection{Neighborhood structures}

ILS $_{\mathrm{RCC}}$ uses three neighborhood structures in the local search, namely: Insertion, Swap and Split. In the following, each of them is described in detail.

\subsubsection{Insertion}

The Insertion neighborhood moves a vertex from a cluster to another one, thus yielding $\mathcal{O}\left(l^{2} n\right)$ possible neighbor solutions to be evaluated.

Algorithm 3 describes how an Insertion move is evaluated using the ADSs. This algorithm receives as input the solution $P$ along with its associated cost (relaxed imbalance) $R I_{P}$, and the information regarding the move, i.e. $S_{p}, i \in S_{p}$ and $S_{q}$. At first, auxiliary variables $\operatorname{sum}_{S_{p}}^{+}, \operatorname{sum}_{S_{p}}^{-}, s_{m_{p}, S_{q}}^{+}$and $s u m_{S_{p}, S_{q}}^{-}$temporarily store, in $\mathcal{O}(1)$ steps, the sum of the weights associated to the move (lines 2-13). Next, the value of the objective function of the neighbor solution under evaluation, denoted in the algorithm as cost, is partially obtained (lines 14-17) by recomputing the penalty decisions using function UpdateCost (see lines 31-36). In the loop from lines 18 to 30, a similar procedure is performed for the intercluster cases involving the other clusters and the clusters $S_{p}$ and $S_{q}$. Finally, cost is returned and the move yields an improvement if cost $<R I_{P}$. 


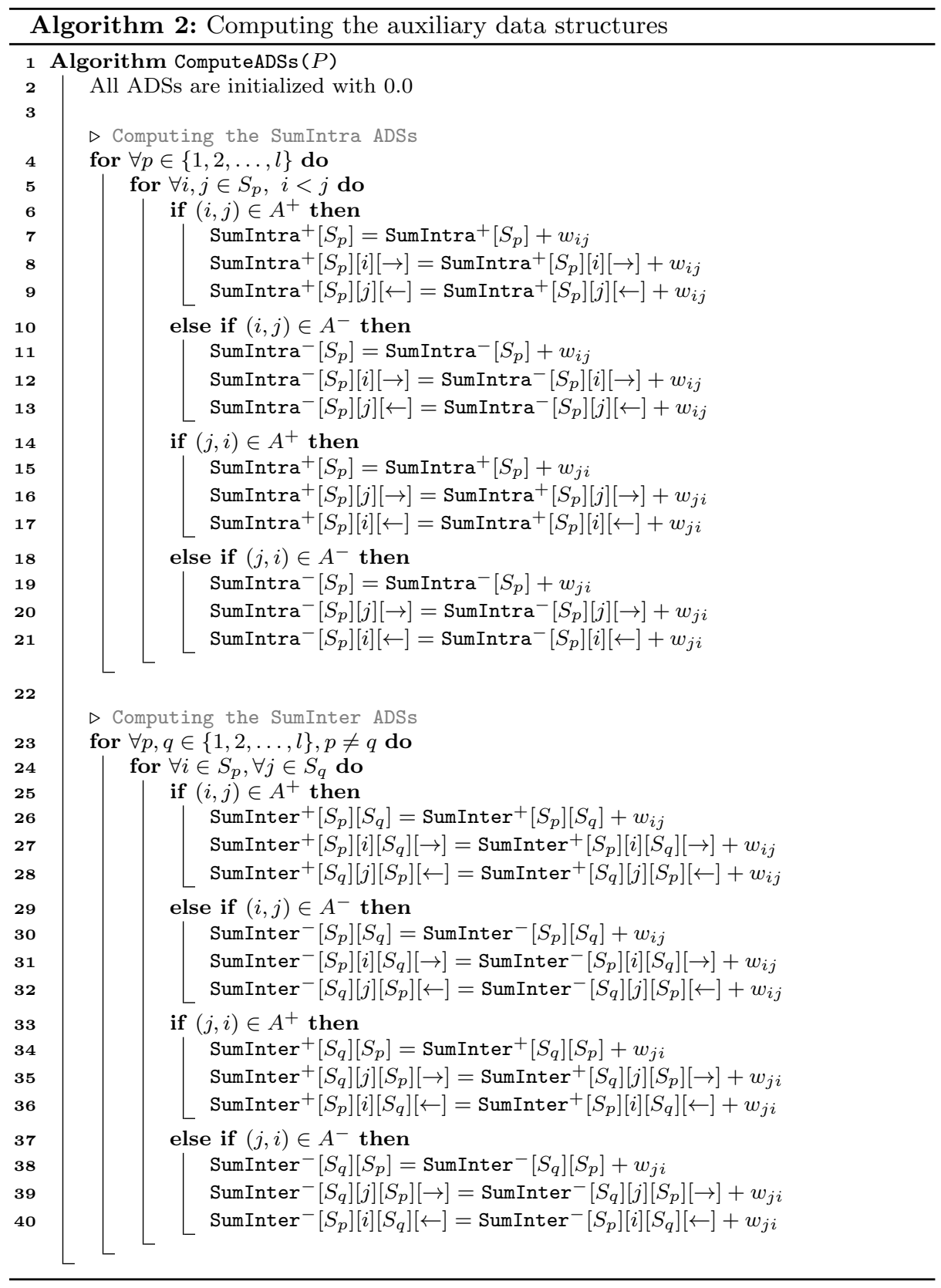


Because Algorithm 3 performs $\mathcal{O}(l)$ steps (due to the loop), finding the best improving move requires $\mathcal{O}\left(l^{3} n\right)$ operations. Moreover, when $P$ is modified, the ADSs must be updated. However, instead of recomputing the ADSs from scratch in $\mathcal{O}\left(l^{2} n^{2}\right)$ operations, one only needs to update the ADSs affected by the vertex that was involved in the move and this can be performed in $\mathcal{O}(n)$ steps, as detailed in the electronic supplementary material of this work.

Figure 3 illustrates an example of an Insertion move. Note that the separation of the weights for the adjacent arcs of vertex $i$ in SumIntra clearly facilitates the evaluation of the intercluster sums from $S_{1}$ to $S_{2}$ and from $S_{2}$ to $S_{1}$. Otherwise, it would be necessary to perform $\mathcal{O}(n)$ operations to compute the weights separately.

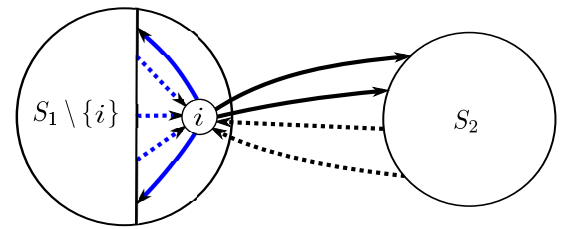

(a) Clusters before Insertion

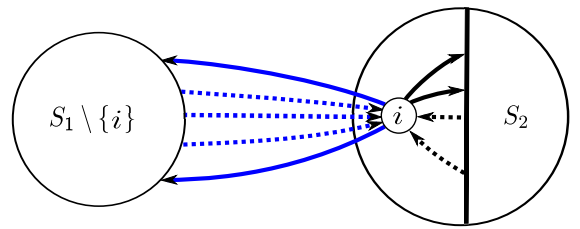

(b) Clusters after Insertion

Fig. 3: Example of an Insertion move. The incoming arcs of $i$ are in dashed lines to illustrate the separation of the weights in SumIntra and SumInter. For the sake of simplicity, the signs were omitted and all arcs have unitary weight.

\subsubsection{Swap}

The Swap neighborhood exchanges two vertices between two different clusters, which leads to $\mathcal{O}\left(l^{2} n^{2}\right)$ neighbor solutions if one intends to enumerate all possibilities. The pseudocodes presented in the electronic supplementary material describe how a $S w a p$ move can be evaluated in $\mathcal{O}(l)$ steps using a similar rationale employed in the Insertion neighborhood. Finding the best improving Swap move thus require $\mathcal{O}\left(l^{3} n^{2}\right)$ operations and the ADSs can be updated in $\mathcal{O}(n)$ steps as also described in the supplementary material.

Figure 4 depicts an example of a swap move, highlighting the arcs connecting exchanged vertices, as they must be treated separately with respect to some ADSs.

\subsubsection{Split}

The Split neighborhood splits a cluster into two, resulting in a total of $\mathcal{O}(\ln )$ neighbor solutions to be examined. Formally, given a cluster $S=\left\{v_{1}, v_{2}, \ldots, v_{|S|}\right\} \in P$ and an index $c<|S|$, the clusters $S^{\prime}=\left\{v_{1}, v_{2}, \ldots, v_{c}\right\}$ and $S^{\prime \prime}=\left\{v_{c+1}, \ldots, v_{|S|}\right\}$ are produced to replace $S$ in $P$. Clearly, a Split move can only be applied when $l<k$. The Pseudocodes presented in the electronic supplementary material describes how a Split move can use previous evaluations to speedup the next ones. The overall complexity of determining the best improvement is $\mathcal{O}\left(\ln ^{2}\right)$, as also 


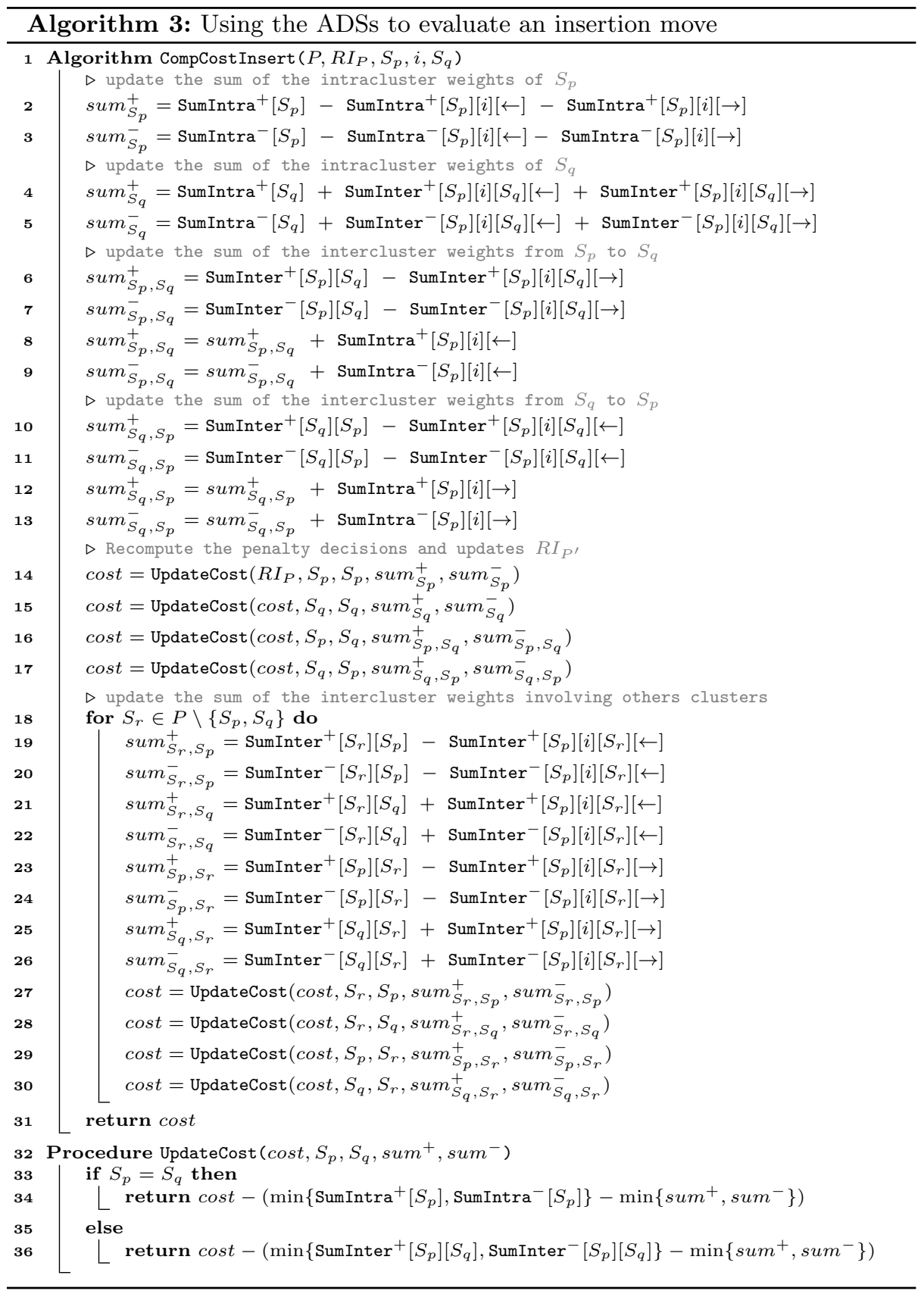

described in the supplementary material. Because of the considerable changes produced by the split move, all ADSs must be updated from scratch using Algorithm 2. It is worth mentioning that implementing a specific procedure to update the ADSs did not pay off the gains in CPU time. Moreover, note that a split move 


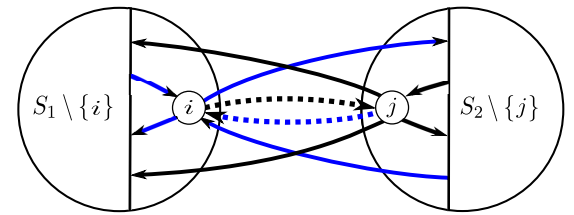

(a) Clusters before Swap

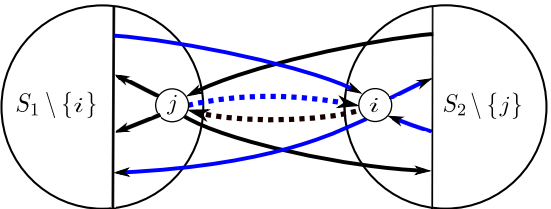

(b) Clusters after Swap

Fig. 4: Example of a Swap move. Arcs $(i, j)$ and $(j, i)$ are in dashed lines to illustrate their importance w.r.t. the ADSs. For the sake of simplicity, the signs were omitted and all arcs have unitary weight.

never worsens a solution, since the imbalance decreases monotonically as $k$ increases [20]. Figure 5 shows an example of a split move considering a cluster with 5 vertices that is split into two with 2 and 3 vertices, respectively.

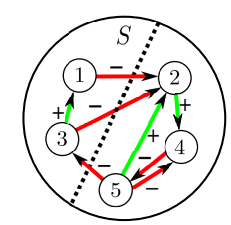

(a) Cluster before Split

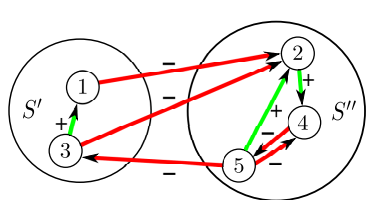

(b) Cluster after Split

Fig. 5: Example of a Split move. For the sake of simplicity, all arcs have unitary weight.

\subsubsection{Complexity summary}

A summary on the complexity of the neighborhoods is provided in Table 3. For each neighborhood, we present its size, as well as the complexity of performing the move evaluation and the overall one using both the efficient best improvement (EBI) and the naive best improvement (NBI) strategies. In EBI, the search for the best improvement move is carried out as described in Section 5.2, whereas in NBI the objective function must be computed from scratch (with no support of ADSs) after each move. We also report the complexity of updating the ADSs in the case of EBI.

Table 3: Complexity summary of the neighborhoods considering both EBI and NBI strategies

\begin{tabular}{|c|c|c|c|c|c|c|}
\hline \multirow{2}{*}{ Neighborhood } & \multirow{2}{*}{ ize } & \multicolumn{3}{|c|}{ EBI } & \multicolumn{2}{|c|}{ NBI } \\
\hline & & Move eval. & Overall & Update & Move eval. & $\mathrm{Ov}$ \\
\hline & & $\mathcal{O}(l)$ & $\mathcal{O}\left(l^{3} n\right)$ & $\mathcal{O}(n)$ & $\mathcal{O}\left(l^{2} n^{2}\right)$ & 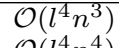 \\
\hline & $\mathcal{O}\left(l^{2} n^{2}\right)$ & $(l)$ & $\mathcal{O}\left(l^{3} n^{2}\right)$ & $\mathcal{O}(n)$ & $\mathcal{O}\left(l^{2} n^{2}\right)$ & $U(l n$ \\
\hline Split & $\mathcal{O}(\ln )$ & $\mathcal{O}(n)$ & $\mathcal{O}\left(\ln ^{2}\right)$ & $\mathcal{O}\left(\ln ^{2}\right)$ & $\mathcal{O}\left(l^{2} n^{2}\right)$ & $\mathcal{O}\left(l^{3} r\right.$ \\
\hline
\end{tabular}




\subsection{Perturbation mechanisms}

ILS $_{\mathrm{RCC}}$ employs three diversification mechanisms to perturb local optimal solutions, namely: Insertion, Merge and Sign inversion. The first one simply performs random insertion moves. In the second, given two clusters $S_{1}$ and $S_{2}$ chosen at random, one merges them to form a new cluster $S_{3}$, that is, $S_{3}=S_{1} \cup S_{2}$. The latter perturbation is a novel procedure that considers some RCC specific features, as described in the following.

The proposed Sign inversion mechanism enforces the penalized sign in one of the decisions to be changed. More precisely, it modifies the solution in such a way that one of the intracluster or intercluster imbalances becomes defined by the opposite sign. The procedure randomly selects which case (i.e., intracluster or intercluster) is going to be considered. Basically, this is achieved by removing vertices that contribute with the non-penalized sign until the inversion happens. In what follows, we will explain the procedure used to invert an intracluster decision.

Let + be the non-penalized sign for the intracluster imbalance of $S_{p}$ (this also applies for $\operatorname{sign}-)$. Formally, the contribution of a vertex $i \in S_{p}$ is given by Equation (20).

$$
\Delta^{+}(i)=\Omega^{+}\left(\{i\}, S_{p}\right)+\Omega^{+}\left(S_{p},\{i\}\right)-\Omega^{-}\left(\{i\}, S_{p}\right)-\Omega^{-}\left(S_{p},\{i\}\right)
$$

At first, the vertices for which all incident arcs (indegree and outdegree arcs) are positive are removed in non-increasing order of $\Delta^{+}$. The value of $\Delta^{+}$must be updated after each removal. If this does not suffice, the remaining vertices with $\Delta^{+}>0$ are removed using the same sorting criterion. Removals are performed while (i) $\Omega^{+}\left(S_{p}, S_{p}\right) \geq \Omega^{-}\left(S_{p}, S_{p}\right)$, (ii) there are vertices with $\Delta^{+}>0$ and (iii) $\left|S_{p}\right|>2$. The removed vertices are randomly added to the other clusters. After applying the perturbation, if $\Omega^{+}\left(S_{p}, S_{p}\right) \geq \Omega^{-}\left(S_{p}, S_{p}\right)$ (i.e. the sign was not inverted), then the removals are undone and the solution returns to the initial state. Figure 6 illustrates an example involving the application of the sign inversion mechanism.

The intercluster sign invertion, e.g., from $S_{p}$ to $S_{q}$, may be easily derived by considering only the $\operatorname{arcs} A\left[S_{p}: S_{q}\right]$ that determine the vertices to be removed from $S_{p}$ and by changing the condition (iii) to $\left|S_{p}\right|>1$. Note that no vertices are removed from $S_{q}$ but it may receive vertices from $S_{p}$.

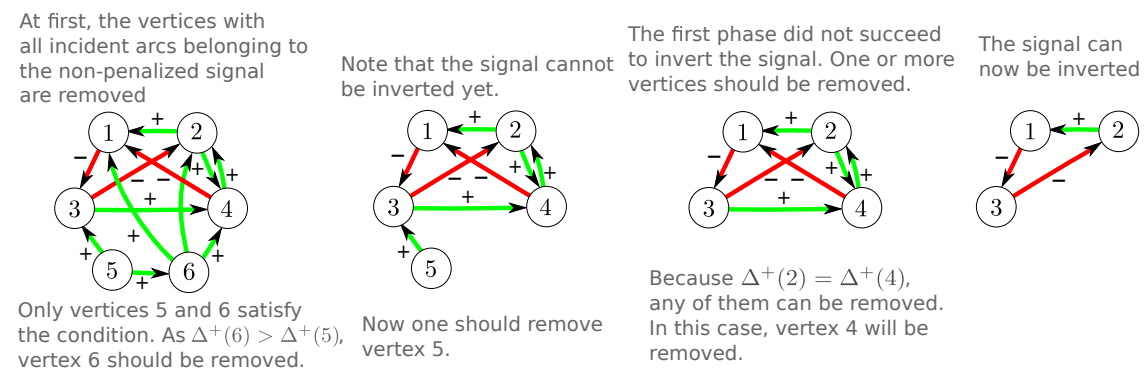

Fig. 6: An example of sign inversion for an intracluster imbalance. For the sake of simplicity, all arcs have unitary weight. 
This perturbation allows for exploring some particular regions of the search space that is difficult to be achieved by only using the other mechanisms, including the randomized construction procedure, mainly when sign distribution on arcs is unbalanced and small changes are not likely to invert the sign.

Each time the function Perturb is called, a perturbation mechanism is randomly chosen. The selected perturbation then applies from two up to maxPert moves. The number of moves is also chosen at random. If $l=2$, Merge is not an eligible perturbation. Therefore, when Sign inversion is chosen and no change could be performed, one of the other two remaining mechanisms (or Insertion if $l=2$ ) is randomly selected.

\subsection{Differences between ILSRCC and ILS [36]}

Table 4 presents the main differences between ILSRCC and the ILS by Levorato et al. [36] which was developed for the SRCC.

Table 4: Differences between ILSRCC and ILS [36]

\begin{tabular}{c|c|c}
\hline & ILS $\mathrm{RCC}$ & ILS [36] \\
\hline Initial solution & Random & Greedy randomized procedure \\
Local search & Insertion, Swap and Split & Insertion \\
Perturbation & Best improvement strategy & First improvement strategy \\
\hline
\end{tabular}

It is worth mentioning that we tried to incorporate the constructive procedure implemented in Levorato et al. [36] into our algorithm, but the experiments reported in Section 6.3.1 indicated that its inclusion did not seem to significantly affect the overall performance of ILS $\mathrm{RCC}_{\mathrm{RC}}$ both in terms of solution quality and CPU time.

\section{Computational results}

All algorithms have been implemented in $\mathrm{C}++$ and executed using a single thread on a PC Intel Core i7-2600 with $3.40 \mathrm{GHz}$ and $16 \mathrm{~GB}$ of RAM running Ubuntu 16.04 LTS (64 bits). For results based on ILP formulations, CPLEX 12.7 is used as a MIP solver (single thread) with all other parameters set to their default values.

\subsection{Benchmark instances}

Regarding the benchmark instances used in our testing, we first present the smallsize instances from the literature. Next, we introduce the newly proposed RCC instances and, finally, we describe the existing SRCC instances. 


\subsubsection{Small-size instances from the literature}

The small-size instances considered here were proposed in different works and together they compose a set of nine signed digraphs described as follows.

- House instances - In 1952, Lemann and Solomon [32] carried out a sociometric study with students living in three different dormitories (denoted as House A, House B and House C) and obtained four relationship networks per dormitory considering the following information: date, friend, roommate and weekend. Doreian [18] later summed the arc weights of the signed networks associated with each dormitory so as to generate another three networks: House A Sum, House B Sum and House C Sum. We have considered these last three in our experiments.

- Monastery instances - In 1868, Sampson [42] studied, in different periods of time, groups of young or novice postulants of a monastery, cataloging data for four types of relationships: affect, esteem, influence, and sanction. From this data, networks were generated for each period of time and type of relationship. Among them, we considered those associated with the relationship affect for different periods of time, namely MonkT2, MonkT3, and MonkT4. In addition, we considered the network Mont4 Sum, generated in Doreian [18] by summing up the arc weights of the four types of relationships in period T4.

- McKinney instance — This signed digraph was built by Brusco et al. [9] from the data collected by McKinney [39] in a study about the relationship between children in a classroom. In such study, children were submitted to a test in which they had to choose between the "willing to serve with other children" (labeled as +1), "not being willing to serve" (labeled as -1) and "indifferent" (labeled as 0), defining the class relationship digraph.

- NewComb instance - In 1961, Newcomb [41] conducted a well-known sociometric study with University students. A signed digraph was generated in Doreian and Mrvar [20] by slightly modifying the data from this study.

The main characteristics of the aforementioned instances are described in Table 5 , where $d$ and $d^{-}$indicate the digraph density (given by $d=|A| /\left(|V|^{2}-|V|\right.$ )) and the percentage of negative arcs (given by $d^{-}=\left|A^{-}\right| /|A|$ ), respectively. For the sake of convenience, we have specified an alias (in parentheses) for each instance.

Table 5: Small-size instance attributes

\begin{tabular}{ccccc}
\hline Name & $|V|$ & $d$ & $d^{-}$ & Author(s) \\
\hline House A Sum (HAS) & 21 & 0.50 & 0.56 & Lemann and Solomon [32], Doreian [18] \\
House B Sum (HBS) & 17 & 0.59 & 0.52 & Lemann and Solomon [32], Doreian [18] \\
House C Sum (HCS) & 20 & 0.52 & 0.53 & Lemann and Solomon [32], Doreian [18] \\
MonkT2 (MT2) & 18 & 0.34 & 0.47 & Sampson [42] \\
MonkT3 (MT3) & 18 & 0.34 & 0.46 & Sampson [42] \\
MonkT4 (MT4) & 18 & 0.34 & 0.46 & Sampson [42] \\
MonkT4 Sum (MT4S) & 18 & 0.50 & 0.49 & Sampson [42], Doreian [18] \\
McKinney (MK) & 18 & 0.34 & 0.10 & McKinney [39], Brusco et al. [9] \\
NewComb (NC) & 17 & 0.44 & 0.43 & Newcomb [41], Doreian and Mrvar [20] \\
\hline
\end{tabular}




\subsubsection{Random instances}

In order to test the ILS implementations on larger instances, we have generated 48 new signed digraphs with different values of $|V|, d$ and $d^{-}$. Let $\mathcal{V}_{|V|}=$ $\{100,200,400,600\}, \mathcal{V}_{d}=\{0.1,0.2,0.5,0.8\}$ and $\mathcal{V}_{d^{-}}=\{0.1,0.3,0.5\}$ be the set of values associated with $|V|, d$ and $d^{-}$, respectively. For each setting obtained by the Cartesian product $\mathcal{V}_{|V|} \times \mathcal{V}_{d} \times \mathcal{V}_{d^{-}}$(represented by a 3-tuple), we have randomly built a signed digraph. Note that larger values of $d^{-}$are not used because they are equivalent with respect to the desired sign distribution (e.g., if $d^{-}=0.7$, then the percentage of positive arcs will be 0.3). A RCC instance consists of a digraph and a value for the parameter $k$ (maximum number of clusters). For each generated digraph, we consider one instance for each value of $k$ in $\{3,5,7,9\}$. Therefore, this benchmark is composed of 192 instances.

The newly generated digraphs are available at http://www.ic.uff.br/ yuri/ files/rcc_random.zip.

\subsubsection{Symmetric RCC instances}

We also considered three sets of symmetric RCC benchmark instances, namely:

- UNGA instances - Generated by Levorato et al. [35] and composed of 63 undirected graphs that were built from the voting data of the United Nations General Assembly (UNGA) annual meetings between 1946 and 2008. These networks are weighted versions of UNGA signed digraphs created by Figueiredo and Frota [25].

- Slashdot instances - Created by Levorato [33] from subgraphs of the social network Slashdot Zoo containing 200 to 10000 vertices. Such subgraphs were transformed into undirected graphs. Levorato [33] performed experiments with a parallel heuristic for the SRCC. Since we are specifically interested in comparing the performance of sequential implementations, it was thought advisable to consider the instances with up to 2000 vertices.

- BR Congress instances - Set of undirected graphs generated by Levorato and Frota [34] from voting sessions of the lower house of Brazilian National Congress. They created two graphs per year between 2011 and 2016, resulting in a total of 12 instances.

The reader is referred to Figueiredo and Frota [25], Levorato et al. [35], Levorato [33], Levorato and Frota [34] for a more detailed description.

\subsection{Results for the ILP formulations}

Tables 6,7 and 8 present the results obtained by the formulation proposed in Figueiredo and Moura [26], as well as those determined by F1 and F2. Column $z$ represents the relaxed imbalance, given by $R I\left(P^{*}\right)$, where $P^{*}$ is the solution (optimal or not) found by the corresponding formulation, gap informs percentage gaps calculated between best integer solutions found and final lower bounds (LB) as described in Equation (21), $\boldsymbol{t}$ indicates the CPU time in seconds ("-" means the instance was not solved in the time limit set), and nodes is the number of nodes that were solved during the search. Regarding the ILP formulation proposed by 
Figueiredo and Moura [26], we report the original results which were found using XPRESS 21.01.00 (Intel Core 2 Duo 2.10 GHz and $3 \mathrm{~GB}$ of RAM) and also those determined by CPLEX 12.7 in order to perform a fair comparison. A time limit of 3600 seconds was imposed for each run.

$$
\text { gap }=100 \times(\text { BestInteger }- \text { LB }) / \text { BestInteger }
$$

We followed the same procedure adopted in Figueiredo and Moura [26] in our testing. For each digraph, we start the experiments with $k=2$. If the instance is solved to optimality, we then increase the value of $k$ by one unit and attempt to solve the problem again (forward phase). If an optimal solution with relaxed imbalance 0 is found, we then interrupt the experiments for that particular digraph since this solution is also optimal for instances with larger values of $k$. When an instance is not solved to optimality, a similar procedure is carried out to solve instances from $k=n-1$, where the value of $k$ is decreased by one unit after each successful optimization (backward phase). In the backward phase, we use the value of the optimal solution found in the previous run (i.e., for $k+1$ ) as a lower bound for the instance with $k$. The backward phase is finished when an instance is not solved to optimality or when the current instance was solved during the forward phase.

Table 6: Results obtained for instances House A Sum, House B Sum and House C Sum.

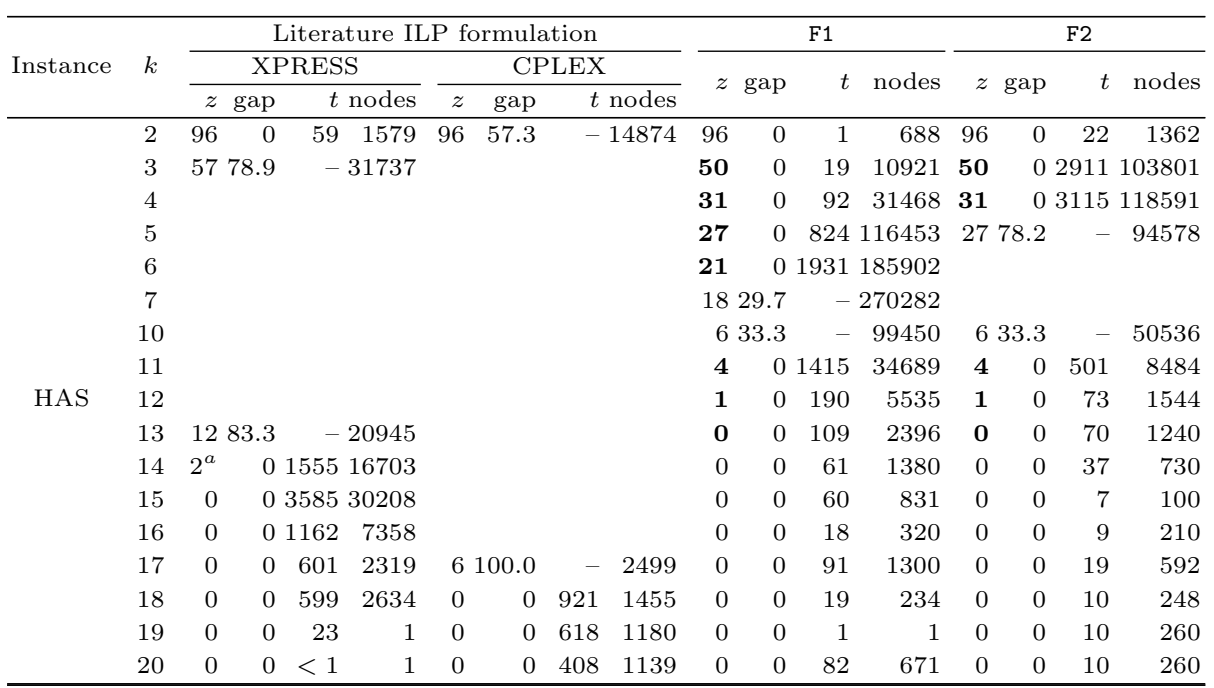


Table 6 - Continued from previous page

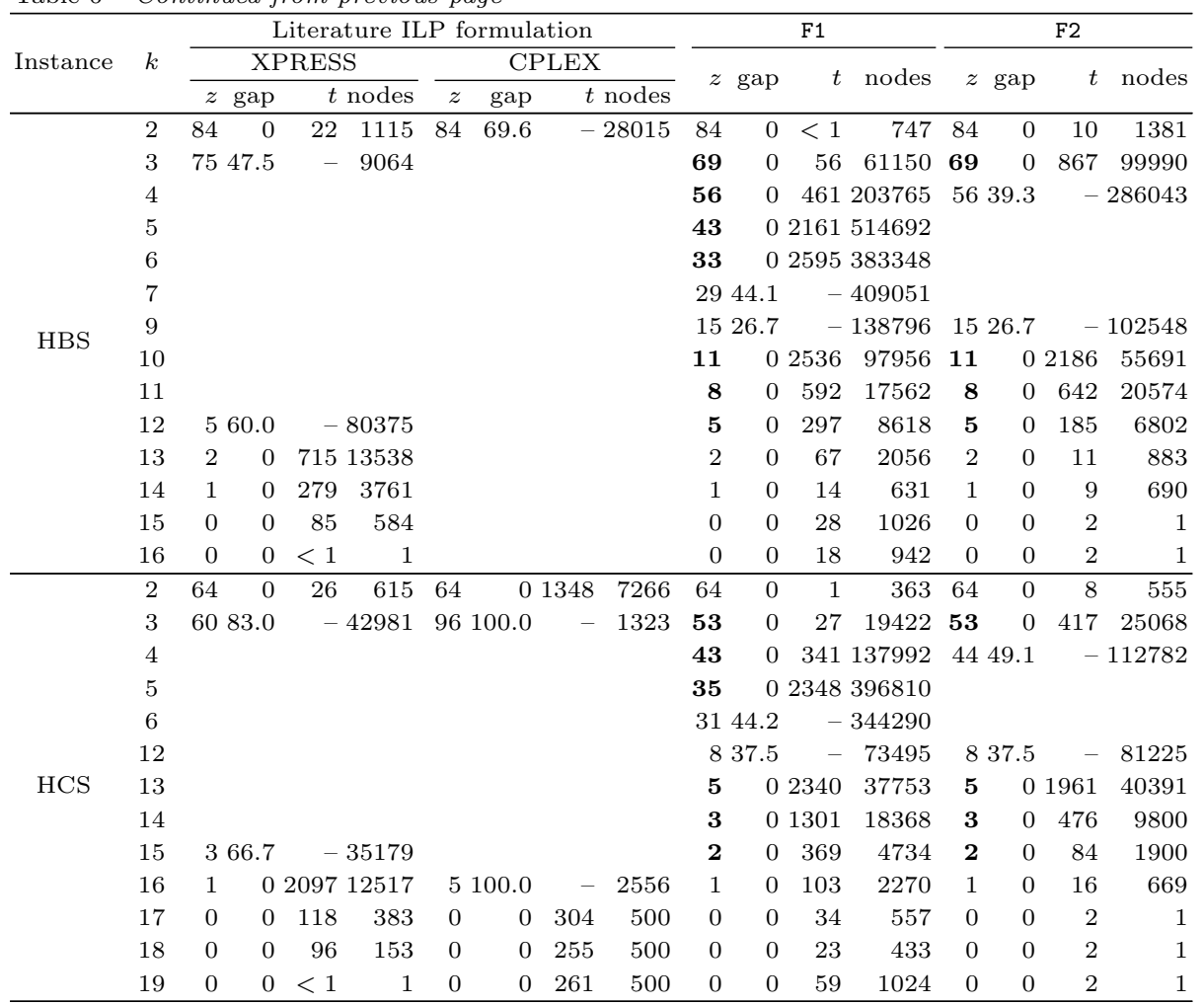

${ }^{a}$ Possible typo

Table 7: Results obtained for instances MonkT2, MonkT3, MonkT4, and MonkT4 Sum.

\begin{tabular}{|c|c|c|c|c|c|c|c|c|c|c|c|c|c|c|c|c|c|}
\hline \multirow{3}{*}{ Instance } & \multirow{3}{*}{$k$} & \multicolumn{8}{|c|}{ Literature ILP formulation } & \multicolumn{4}{|c|}{ F1 } & \multicolumn{4}{|c|}{ F2 } \\
\hline & & \multicolumn{4}{|c|}{ XPRESS } & \multicolumn{4}{|c|}{ CPLEX } & \multirow{2}{*}{\multicolumn{2}{|c|}{$z$ gap }} & \multirow{2}{*}{\multicolumn{2}{|c|}{$t$ nodes }} & \multirow{2}{*}{\multicolumn{2}{|c|}{$z$ gap }} & \multirow{2}{*}{\multicolumn{2}{|c|}{$t$ nodes }} \\
\hline & & $z$ & gap & $t$ & nodes & $z$ & gap & & $\overline{\text { nodes }}$ & & & & & & & & \\
\hline \multirow{16}{*}{ MT2 } & 2 & 43 & 0 & 13 & 733 & 43 & 0 & 37 & 1343 & 43 & 0 & $<1$ & 818 & 43 & 0 & 2 & 534 \\
\hline & 3 & 25 & 0 & 2238 & 70771 & 27 & 92.6 & & 11388 & 25 & 0 & 5 & 7049 & 25 & 0 & 42 & 6271 \\
\hline & 4 & 20 & 85.0 & & 121561 & & & & & 13 & 0 & 9 & 6998 & 13 & 0 & 212 & 26725 \\
\hline & 5 & & & & & & & & & 8 & 0 & 15 & 7077 & 8 & 0 & 116 & 11601 \\
\hline & 6 & & & & & & & & & 4 & 0 & 11 & 3765 & 4 & 0 & 76 & 8493 \\
\hline & 7 & & & & & 14 & 92.9 & & 12097 & 2 & 0 & 4 & 1177 & 2 & 0 & 6 & 718 \\
\hline & 8 & & & & & 1 & 0 & 509 & 2497 & 1 & 0 & 2 & 236 & 1 & 0 & 4 & 488 \\
\hline & 9 & & & & & 0 & 0 & 666 & 3835 & 0 & 0 & 1 & 252 & 0 & 0 & 4 & 720 \\
\hline & 10 & & & & & 0 & 0 & 128 & 1484 & & & & & & & & \\
\hline & 11 & \multicolumn{3}{|c|}{2100.0} & 176063 & 0 & 0 & 169 & 1781 & & & & & & & & \\
\hline & 12 & 0 & 0 & 2369 & 102937 & 0 & 0 & 80 & 1578 & & & & & & & & \\
\hline & 13 & 0 & 0 & 222 & 8881 & 0 & 0 & 19 & 278 & & & & & & & & \\
\hline & 14 & 0 & 0 & 48 & 593 & 0 & 0 & 54 & 1438 & & & & & & & & \\
\hline & 15 & 0 & 0 & 15 & 69 & 0 & 0 & 62 & 1543 & & & & & & & & \\
\hline & 16 & 0 & 0 & 7 & 1 & 0 & 0 & 61 & 1597 & & & & & & & & \\
\hline & 17 & 0 & 0 & $<1$ & 1 & 0 & 0 & 40 & 1082 & & & & & & & & \\
\hline
\end{tabular}




\begin{tabular}{|c|c|c|c|c|c|c|c|c|c|c|c|c|c|c|c|c|c|}
\hline \multirow{3}{*}{ Instance } & \multirow{3}{*}{$k$} & \multicolumn{8}{|c|}{ Literature ILP formulation } & \multicolumn{4}{|c|}{ F1 } & \multicolumn{4}{|c|}{ F2 } \\
\hline & & \multicolumn{4}{|c|}{ XPRESS } & \multicolumn{4}{|c|}{ CPLEX } & \multirow{2}{*}{\multicolumn{2}{|c|}{$z$ gap }} & \multirow{2}{*}{\multicolumn{2}{|c|}{$t$ nodes }} & \multirow{2}{*}{\multicolumn{2}{|c|}{$z$ gap }} & \multirow{2}{*}{\multicolumn{2}{|c|}{$t$ nodes }} \\
\hline & & $z$ & gap & $t$ & $t$ nodes & $z \mathrm{~g}$ & sap & & $\overline{\text { nodes }}$ & & & & & & & & \\
\hline \multirow{16}{*}{ MT3 } & 2 & 32 & 0 & 6 & 243 & 32 & 0 & 30 & 1404 & 32 & 0 & $<1$ & 126 & 32 & 0 & 2 & 109 \\
\hline & 3 & 21 & 0 & 193 & 4765 & 2892 & 2.9 & - & 8924 & 21 & 0 & 1 & 876 & 21 & 0 & 7 & 1964 \\
\hline & 4 & 13 & & 2269 & $9 \quad 54227$ & & & & & 13 & 0 & 2 & 1835 & 13 & 0 & 13 & 2690 \\
\hline & 5 & 8 & & 3325 & 585056 & & & & & 8 & 0 & 7 & 3460 & 8 & 0 & 10 & 2013 \\
\hline & 6 & 7 & 71.4 & & -100613 & & & & & 6 & 0 & 4 & 1840 & 6 & 0 & 34 & 3979 \\
\hline & 7 & 5 & 60.0 & & -140597 & 450 & 0.0 & & 21250 & 4 & 0 & 4 & 1551 & 4 & 0 & 9 & 1749 \\
\hline & 8 & 2 & & 2837 & 6725 & 2 & 0 & 675 & 6642 & 2 & 0 & 2 & 517 & 2 & 0 & 5 & 739 \\
\hline & 9 & 1 & 0 & 438 & $\begin{array}{ll}8 & 11577\end{array}$ & 1 & 0 & 56 & 1610 & 1 & 0 & 1 & 171 & 1 & 0 & 3 & 290 \\
\hline & 10 & 0 & & 1182 & $2 \quad 44984$ & 0 & 0 & 50 & 1090 & 0 & & $<1$ & 1 & 0 & 0 & 2 & 402 \\
\hline & 11 & 0 & 0 & 251 & 8439 & 0 & 0 & 32 & 989 & & & & & & & & \\
\hline & 12 & 0 & 0 & 519 & $9 \quad 21543$ & 0 & 0 & 7 & 150 & & & & & & & & \\
\hline & 13 & 0 & 0 & 103 & 5335 & 0 & 0 & 7 & 143 & & & & & & & & \\
\hline & 14 & 0 & 0 & 49 & 1120 & 0 & 0 & 8 & 174 & & & & & & & & \\
\hline & 15 & 0 & 0 & 30 & 455 & 0 & 0 & 8 & 197 & & & & & & & & \\
\hline & 16 & 0 & 0 & 18 & 119 & 0 & 0 & 8 & 197 & & & & & & & & \\
\hline & 17 & 0 & 0 & $<1$ & 1 & 0 & 0 & 8 & 197 & & & & & & & & \\
\hline \multirow{16}{*}{ MT4 } & 2 & 25 & 0 & 5 & 149 & 25 & 0 & 11 & 206 & 25 & 0 & $<1$ & 194 & 25 & 0 & 1 & 99 \\
\hline & 3 & 21 & 0 & 119 & 3381 & 2175 & 5.2 & & 12524 & 21 & 0 & 1 & 923 & 21 & 0 & 6 & 1459 \\
\hline & 4 & 10 & 0 & 563 & 313945 & & & & & 10 & 0 & 1 & 706 & 10 & 0 & 9 & 1864 \\
\hline & 5 & 6 & & 1463 & 342782 & & & & & 6 & 0 & 3 & 1283 & 6 & 0 & 64 & 6038 \\
\hline & 6 & 4 & 0 & 2391 & $1 \quad 68659$ & 580 & 0.0 & - & 7519 & 4 & 0 & 2 & 719 & 4 & 0 & 47 & 4389 \\
\hline & 7 & 1 & 0 & 683 & 319452 & 1 & 0 & 141 & 1370 & 1 & 0 & 1 & 308 & 1 & 0 & 3 & 476 \\
\hline & 8 & 0 & 0 & 414 & $4 \quad 12469$ & 0 & 0 & 31 & 691 & 0 & 0 & 1 & 61 & 0 & 0 & 2 & 331 \\
\hline & 9 & & & & & 0 & 0 & 66 & 537 & & & & & & & & \\
\hline & 10 & & & & & 0 & 0 & 35 & 756 & & & & & & & & \\
\hline & 11 & & & & & 0 & 0 & 4 & 1 & & & & & & & & \\
\hline & 12 & & & & & 0 & 0 & 11 & 1 & & & & & & & & \\
\hline & 13 & & & & & 0 & 0 & 11 & 1 & & & & & & & & \\
\hline & 14 & & & & & 0 & 0 & 9 & 1 & & & & & & & & \\
\hline & 15 & & & & & 0 & 0 & 8 & 1 & & & & & & & & \\
\hline & 16 & & & & & 0 & 0 & 9 & 1 & & & & & & & & \\
\hline & 17 & & & & & 0 & 0 & 8 & 1 & & & & & & & & \\
\hline \multirow{16}{*}{ MT4S } & 2 & 86 & 0 & 14 & 347 & 86 & 0 & 66 & 518 & 86 & 0 & $<1$ & 217 & 86 & 0 & 2 & 229 \\
\hline & 3 & 54 & 0 & 1539 & $9 \quad 25379$ & 8598 & 8.8 & - & 2941 & 54 & 0 & 1 & 988 & 54 & 0 & 80 & 5595 \\
\hline & 4 & 43 & 72.2 & - & 7483 & & & & & 36 & 0 & 8 & 3340 & 36 & & 298 & 21034 \\
\hline & 5 & & & & & & & & & 25 & 0 & 23 & 6128 & 25 & & 300 & 17832 \\
\hline & 6 & & & & & & & & & 16 & 0 & 42 & 7039 & 16 & & 225 & 11659 \\
\hline & 7 & & & & & & & & & 12 & 0 & 41 & 5561 & 12 & & 131 & 5068 \\
\hline & 8 & & & & & 966 & 6.7 & - & 9104 & 8 & 0 & 18 & 2281 & 8 & 0 & 58 & 2232 \\
\hline & 9 & 6 & 100.0 & - & - 61861 & 3 & & 3407 & 5188 & 3 & 0 & 7 & 590 & 3 & 0 & 8 & 525 \\
\hline & 10 & 2 & & 2582 & 251491 & 2 & & 3498 & 5701 & 2 & 0 & 3 & 172 & 2 & 0 & 6 & 372 \\
\hline & 11 & 0 & 0 & 1740 & 25094 & 0 & 0 & 79 & 470 & 0 & 0 & 1 & 1 & 0 & 0 & 8 & 602 \\
\hline & 12 & 0 & 0 & 925 & $5 \quad 11838$ & 0 & 0 & 126 & 935 & & & & & & & & \\
\hline & 13 & 0 & 0 & 58 & 240 & 0 & 0 & 108 & 935 & & & & & & & & \\
\hline & 14 & 0 & 0 & 300 & 3389 & 0 & 0 & 18 & 1 & & & & & & & & \\
\hline & 15 & 0 & 0 & 7 & 1 & 0 & 0 & 19 & 1 & & & & & & & & \\
\hline & 16 & 0 & 0 & 1 & 1 & 0 & 0 & 19 & 1 & & & & & & & & \\
\hline & 17 & 0 & 0 & $<1$ & 1 & 0 & 0 & 18 & 1 & & & & & & & & \\
\hline
\end{tabular}


Table 8: Results obtained for instances McKinney and NewComb.

\begin{tabular}{|c|c|c|c|c|c|c|c|c|c|c|c|c|c|c|c|c|c|c|}
\hline \multirow{3}{*}{ Instance } & \multirow{3}{*}{$k$} & \multicolumn{8}{|c|}{ Literature ILP formulation } & \multicolumn{5}{|c|}{ F1 } & \multicolumn{4}{|c|}{ F2 } \\
\hline & & \multicolumn{4}{|c|}{ XPRESS } & \multicolumn{4}{|c|}{ CPLEX } & \multirow{2}{*}{\multicolumn{2}{|c|}{$z$ gap }} & \multirow{2}{*}{\multicolumn{3}{|c|}{$t$ nodes }} & \multirow{2}{*}{\multicolumn{2}{|c|}{$z$ gap }} & \multirow{2}{*}{\multicolumn{2}{|c|}{$t$ nodes }} \\
\hline & & $z$ & gap & $\bar{t}$ & $\overline{\text { nodes }}$ & $z$ & gap & & nodes & & & & & & & & & \\
\hline \multirow{19}{*}{ MK } & 2 & 8 & 0 & 118 & 6531 & 8 & 100.0 & - & 7559 & 8 & 0 & $<$ & 1 & 28 & 8 & & 112 & 2802 \\
\hline & 3 & 6 & 100.0 & - & 43762 & & & & & 2 & 0 & $<$ & 1 & 197 & 2 & & 227 & 7766 \\
\hline & 4 & & & & & & & & & $\mathbf{0}$ & 0 & $<$ & & 1 & 0 & 0 & 50 & 1567 \\
\hline & 13 & & & & & 2 & 100.0 & - & 1432 & & & & & & & & & \\
\hline & 14 & & & & & 0 & 0 & 57 & 1 & & & & & & & & & \\
\hline & 15 & & & & & 0 & 0 & 59 & 1 & & & & & & & & & \\
\hline & 16 & 2 & 100.0 & - & 33562 & 0 & 0 & 53 & 1 & & & & & & & & & \\
\hline & 17 & 0 & 0 & 81 & 169 & 0 & 0 & 53 & 1 & & & & & & & & & \\
\hline & 18 & 0 & 0 & 2 & 1 & 0 & 0 & 52 & 1 & & & & & & & & & \\
\hline & 19 & 0 & 0 & 19 & 1 & 0 & 0 & 53 & 1 & & & & & & & & & \\
\hline & 20 & 0 & 0 & 1 & 1 & 0 & 0 & 55 & 1 & & & & & & & & & \\
\hline & 21 & 0 & 0 & 16 & 1 & 0 & 0 & 58 & 1 & & & & & & & & & \\
\hline & 22 & 0 & 0 & 2 & 1 & 0 & 0 & 55 & 1 & & & & & & & & & \\
\hline & 23 & 0 & 0 & 5 & 1 & 0 & 0 & 60 & 1 & & & & & & & & & \\
\hline & 24 & 0 & 0 & 6 & 1 & 0 & 0 & 58 & 1 & & & & & & & & & \\
\hline & 25 & 0 & 0 & 1 & 1 & 0 & 0 & 53 & 1 & & & & & & & & & \\
\hline & 26 & 0 & 0 & 95 & 49 & 0 & 0 & 57 & 1 & & & & & & & & & \\
\hline & 27 & 0 & 0 & 2 & 1 & 0 & 0 & 59 & 1 & & & & & & & & & \\
\hline & 28 & 0 & 0 & $<1$ & 1 & 0 & 0 & 54 & 1 & & & & & & & & & \\
\hline \multirow{15}{*}{$\mathrm{NC}$} & 2 & 10 & 0 & 4 & 167 & 10 & 0 & 34 & 468 & 10 & 0 & $<$ & 1 & 66 & 10 & 0 & 1 & 101 \\
\hline & 3 & 7 & 0 & 475 & 9869 & \multirow{2}{*}{\multicolumn{2}{|c|}{8100.0}} & - & 4988 & 7 & 0 & & 1 & 1193 & 7 & 0 & 43 & 6188 \\
\hline & 4 & 5 & 34.6 & - & 90604 & & & & & 5 & 0 & & 7 & 5570 & 5 & 0 & & 10538 \\
\hline & 5 & & & & & 6 & 83.3 & - & 9349 & 3 & 0 & 1 & 1 & 4690 & 3 & 0 & 80 & 8054 \\
\hline & 6 & & & & & 1 & & 1299 & 6295 & 1 & 0 & & 1 & 331 & 1 & 0 & 35 & 3561 \\
\hline & 7 & & & & & 0 & & 2646 & 7542 & 0 & 0 & & 3 & 1093 & 0 & 0 & 12 & 1177 \\
\hline & 8 & 1 & 100.0 & - & 146619 & 0 & 0 & 846 & 2861 & & & & & & & & & \\
\hline & 9 & 0 & & 172 & 9807 & 0 & 0 & 594 & 2624 & & & & & & & & & \\
\hline & 10 & 0 & 0 & 123 & 5969 & 0 & 0 & 500 & 2276 & & & & & & & & & \\
\hline & 11 & 0 & 0 & 27 & 405 & 0 & 0 & 46 & 1005 & & & & & & & & & \\
\hline & 12 & 0 & 0 & 37 & 162 & 0 & 0 & 142 & 1815 & & & & & & & & & \\
\hline & 13 & 0 & 0 & 8 & 1 & 0 & 0 & 294 & 2075 & & & & & & & & & \\
\hline & 14 & 0 & & $<1$ & 1 & 0 & 0 & 116 & 1172 & & & & & & & & & \\
\hline & 15 & 0 & & $<1$ & 1 & 0 & 0 & 85 & 1505 & & & & & & & & & \\
\hline & 16 & 0 & & $<1$ & 1 & 0 & 0 & 64 & 1505 & & & & & & & & & \\
\hline
\end{tabular}

The results obtained show that $\mathrm{F} 1$ outperforms the other formulations with respect to the number of optimal solutions achieved. When a formulation obtained an optimal solution with relaxed imbalance 0 for a given value of $k$, then we assume that all optima were found for executions with larger values of $k$ (e.g., F1 and F2 solved instances from MT2 to optimality). While this formulation found 38, 64, 27, 15 optimal solutions for each group (House, Monastery, McKinney, NewComb), respectively, F2 found 29, 64, 27, 15, respectively, and the formulation by Figueiredo and Moura [26] obtained 18, 48, 13, 10 using XPRESS, and 7, 44, 15, 12 using CPLEX, respectively. Overall, a total of 40 new optimal solutions (counting only once the optimal solutions with relaxed imbalance 0 for each digraph) were found and all instances of 6 of the 9 groups were solved to optimality.

In addition, it can be observed that F1 is generally faster, but it is outrun by F2 on instances HAS, HBS and HCS for larger values of $k$. Note that for the latter two, F2 solves some instances at the root node. The formulation by Figueiredo 
and Moura [26] is clearly slower, even taking into account the hardware difference for the results found using XPRESS. In general, more nodes are solved when running such formulation, but in some cases, F2 is the one to solve more nodes.

The results also demonstrate that RCC has the expected behavior for $k$ partition problems, where problems with $k$ close to 2 and $n-1$ are easier than those problems with $k$ close to $n / 2$. This can be explained by the number of possible partitions, which is given by the Stirling number of the second type [8] as described in Equation (22).

$$
S(n, k)=\frac{1}{k !} \sum_{j=0}^{k}(-1)^{k-j}\left(\begin{array}{l}
k \\
j
\end{array}\right) j^{n}
$$

Finally, we report in Table 9 a comparison between the optimal solutions for RCC and CC. In addition to comparing $I(P)$ with $R I(P)$ and the correction $\delta=I(P)-R I(P)$ obtained with RCC (recall that RCC was proposed in order to correct wrong penalties in $\mathrm{CC}$ ), we also decompose the total imbalance into minimum, average, and maximum penalties for intracluster and intercluster cases. With the exception of one instance (MT4 with $k=3$ ), a positive correction is obtained by RCC. It is worth mentioning that most of the imbalance (and hence the correction) occurs in intercluster cases.

Table 9: Comparison of optimal solutions for RCC and CC in small instances.

\begin{tabular}{|c|c|c|c|c|c|c|c|c|c|c|c|c|c|c|c|c|}
\hline \multirow{3}{*}{ Name } & \multirow{3}{*}{$k$} & \multicolumn{7}{|c|}{$I(P)$} & \multicolumn{7}{|c|}{$R I(P)$} & \multirow{3}{*}{$\delta$} \\
\hline & & \multirow{2}{*}{ total } & \multicolumn{3}{|c|}{ intra } & \multicolumn{3}{|c|}{ inter } & \multirow{2}{*}{ total } & \multicolumn{3}{|c|}{ intra } & \multicolumn{3}{|c|}{ inter } & \\
\hline & & & $\min$ & avg & $\max$ & $\min$ & avg & $\max$ & & $\min$ & avg & $\max$ & $\min$ & avg & $\max$ & \\
\hline HAS & 4 & 64 & 0 & 4.8 & 16 & 0 & 7.5 & 19 & 31 & 0 & 2.3 & 5 & 0 & 1.8 & 5 & 33 \\
\hline HBS & 4 & 81 & 0 & 3.5 & 14 & 1 & 11.2 & 23 & 56 & 0 & 5.8 & 11 & 0 & 2.8 & 10 & 25 \\
\hline HCS & 3 & 59 & 0 & 5.3 & 10 & 4 & 14.3 & 27 & 53 & 3 & 5.7 & 8 & 3 & 6.0 & 10 & 6 \\
\hline MT2 & 3 & 35 & 0 & 0.7 & 1 & 3 & 11.0 & 16 & 25 & 0 & 0.3 & 1 & 1 & 4.0 & 7 & 10 \\
\hline MT3 & 3 & 22 & 0 & 0.3 & 1 & 4 & 7.0 & 11 & 21 & 0 & 1.0 & 3 & 0 & 3.0 & 7 & 1 \\
\hline MT4 & 3 & 21 & 0 & 1.7 & 5 & 3 & 5.3 & 9 & 21 & 0 & 1.7 & 5 & 0 & 2.7 & 6 & 0 \\
\hline MT4S & 3 & 62 & 1 & 3.0 & 7 & 12 & 17.7 & 25 & 54 & 1 & 3.0 & 7 & 2 & 7.5 & 15 & 8 \\
\hline MK & 2 & 12 & 0 & 3.0 & 6 & 6 & 6.0 & 6 & 8 & 0 & 2.0 & 4 & 2 & 2.0 & 2 & 4 \\
\hline $\mathrm{NC}$ & 4 & 20 & 0 & 1.3 & 5 & 0 & 2.5 & 7 & 5 & 0 & 0.5 & 1 & 0 & 0.3 & 1 & 15 \\
\hline
\end{tabular}

\subsection{ILS implementations}

We used the same values adopted in Subramanian and Farias [45] for the main parameters of ILSRCC, that is, $I_{R}=20$ and $I_{I L S}=\min \{100,4 \times n\}$. The only difference is that we imposed a minimum value of 100 for the latter as in Silva et al. [43]. Moreover, we set $\max P$ ert $=6$ after conducting some experiments (see Section 6.3.1). The algorithms were executed 10 times on each instance in all experiments. Hereafter, the percentage gap of a solution $P^{\prime}$ is computed as gap $=100 \times\left(f\left(P^{\prime}\right)-f\left(P_{\text {best }}\right)\right) / f\left(P^{\prime}\right)$, such that $f$ is the objective function (i.e., $f(P)=R I(P)$ for RCC and $f(P)=S R I(P)$ for SRCC) and $P_{\text {best }}$ is the best

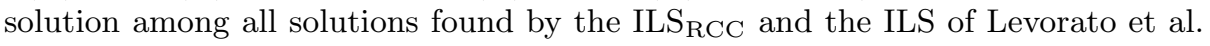
[36]. 


\subsubsection{Impact of the different components of the algorithm}

We evaluate the ILS $\mathrm{RCC}_{\mathrm{RC}}$ concerning the impact of: (i) using the greedy constructive algorithm by Levorato et al. [36]; (ii) the parameter maxPert; (iii) the neighborhood structures; (iv) perturbation mechanisms. To this end, we conducted experiments involving all 100-vertex random instances.

At first, we assess the impact of replacing the completely random construction (line 5 in Algorithm 1) with the greedy construction of Levorato et al. [36]. Table 10 shows the average gaps and CPU times obtained by the two versions of ILS $\mathrm{RCC}$ (using two different constructive procedures) for different values of $k$. It can be seen that none of the two versions are significantly superior than the other w.r.t. both criteria. The only exception occurs when $k=9$, where using the completely random construction produces a superior average gap and CPU time. In general, using the greedy construction in ILS $_{\mathrm{RCC}}$ leads to an improvement of only $0.01 \%$ in terms of average gap, and an increase of around $3 \%$ on the average CPU time. Therefore, it is reasonable to conclude that the effort to implement a more sophisticated constructive procedure may not be worthwhile for the RCC when the algorithm contains an effective local search.

Table 10: Comparison of two constructive heuristics in ILS $_{\mathrm{RCC}}$ for different values of $k$.

\begin{tabular}{cccc}
\hline Construction & $k$ & Avg. gap (\%) & Avg. time (s) \\
\hline \multirow{3}{*}{ Greedy [36] } & 3 & $\mathbf{0 . 1 4}$ & 7.09 \\
& 5 & $\mathbf{0 . 9 2}$ & 13.76 \\
& 7 & $\mathbf{1 . 7 7}$ & 19.78 \\
& 9 & 2.84 & 24.20 \\
\hline \hline Mean & & $\mathbf{1 . 4 2}$ & 16.21 \\
\hline \multirow{2}{*}{ Random } & 3 & 0.17 & $\mathbf{6 . 8 3}$ \\
& 5 & 1.03 & $\mathbf{1 3 . 3 6}$ \\
& 7 & 1.96 & $\mathbf{1 9 . 2 0}$ \\
\hline Mean & 9 & $\mathbf{2 . 5 6}$ & $\mathbf{2 3 . 3 1}$ \\
\hline
\end{tabular}

The impact of varying the parameter maxPert is illustrated in Figure 7, where values between 4 and 8 are considered to compare different versions of ILSRCC. The results show that the values 5 and 7 are dominated by the remaining ones; the value 4 produces the faster execution but with a poor average gap, whereas the value 8 achieves the best average gap but the worst CPU time. Therefore, we decided to use maxPert $=6$ because it yields a good balance between solution quality and CPU time.

We assess the impact of the neighborhood operations by considering 7 different configurations. In this case, we consider $I_{R}=20$ and $I_{I L S}=1$ (i.e. only one iteration of the RVND procedure is executed) and we measure the percentage improvement over the initial solution. The average results are shown in Figure 8. We can observe that the configurations that yields the most promising results are those 5 and 7 . The difference between both settings is that the latter includes the neighborhood Swap, which led to a slight improvement despite the additional CPU time. 


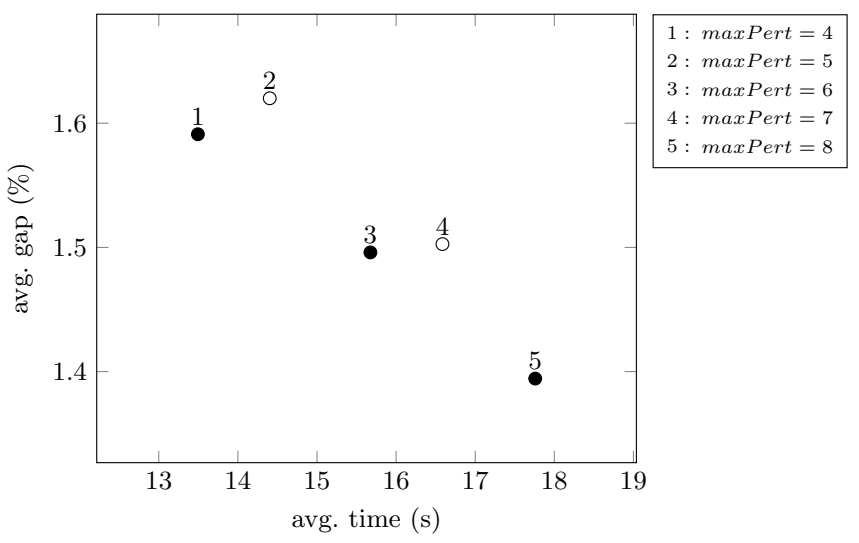

Fig. 7: Impact of the parameter maxPert. Each point represents a configuration and points with no fill represent those dominated by one or more settings.

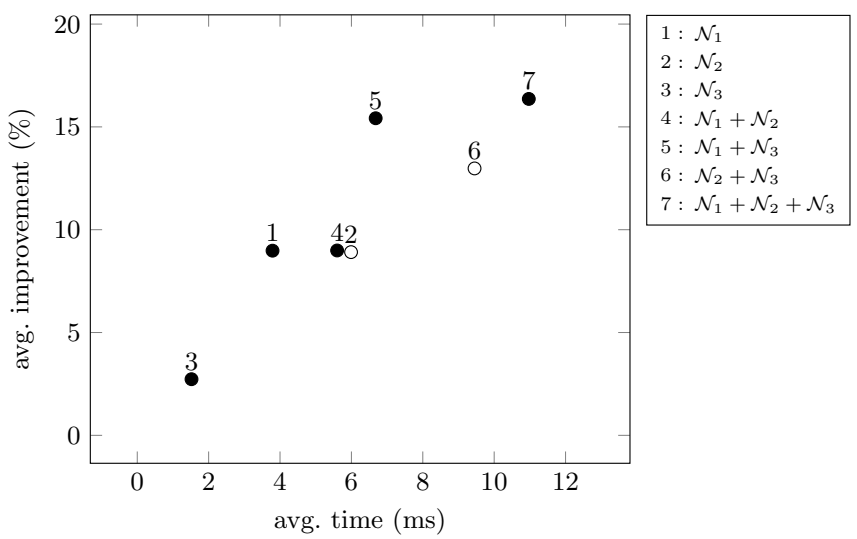

Fig. 8: Impact of the neighborhood operations. Each point represents a configuration and points with no fill represent those dominated by one or more settings. $\mathcal{N}_{1}, \mathcal{N}_{2}$ and $\mathcal{N}_{3}$ denote neighborhoods Insert, Swap and Split, respectively.

In order to measure the impact of the perturbation mechanisms, we run ILS $_{\mathrm{RCC}}$ using the default values of the parameters and store the percentage improvement over the best solution found in the previous testing for each instance. To choose an interesting configuration, we perform experiments considering two scenarios: with and without the neighborhood swap. The average results obtained are depicted in Figure 9. The best results are obtained by settings 6 and 7 for both scenarios. The scenario that considered Swap achieves better improvements at the expense of CPU time. Although not reported in Figure 9, the settings tested in the second scenario (i.e., the one including Swap) systematically found more best solutions than their corresponding counterpart in the first scenario. We, therefore, decided to select configuration 7 of the second scenario because it appears to offer an interesting compromise between solution quality and CPU time. 


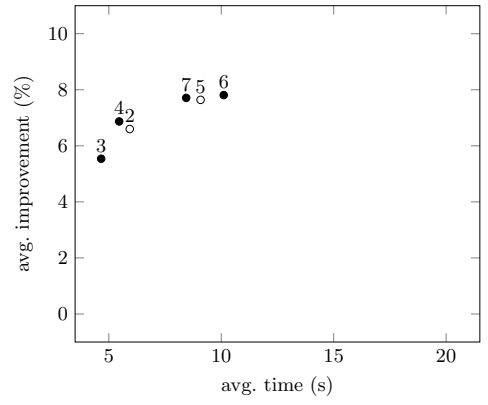

(a)

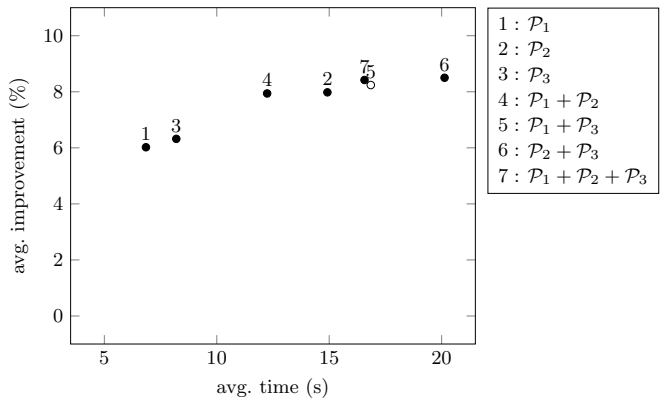

(b)

Fig. 9: Impact of the perturbation operations. Each point represents a configuration and points with no fill represent those dominated by one or more settings. $\mathcal{P}_{1}, \mathcal{P}_{2}$ and $\mathcal{P}_{3}$ denote perturbations Insert, Split and Sign Inversion, respectively. Part (a) does not include the neighborhood Swap whereas part (b) does.

\subsubsection{Comparison with the literature}

The implementation by Levorato et al. [36] was originally devised for the symmetric version of the problem. Therefore, we had to slightly modify the source code, which was provided by the authors, to cope with the asymmetric case. We will refer to this method as ILS adapt $_{\text {. }}$

Concerning the small-size instances considered in Section 6.1.1, it was observed that ILS $_{\mathrm{RCC}}$ and ILS $\mathrm{S}_{\mathrm{adapt}}$ are capable of consistently finding the optimal solutions in a fraction of a second.

Table 11 shows the aggregate results obtained by the ILS implementations on the set of random instances. For each digraph, we report the minimum, average and maximum values of the average percentage gaps (there is an average gap for each value of $k \in\{3,5,7,9\})$, as well as the average CPU time in seconds. Detailed results are reported in Appendix A. Moreover, for an appropriate comparison, we have imposed the average CPU time obtained by $\mathrm{ILS}_{\mathrm{RCC}}$, for each instance, as a stopping criterion for ILS adapt.

Table 11: Aggregate results for each digraph. Each row reports statistics on the average gaps obtained for the group of four instances (one for each value of $k \in$ $\{3,5,7,9\})$.

\begin{tabular}{|c|c|c|c|c|c|c|c|c|c|}
\hline \multirow{2}{*}{$|V|$} & \multirow{2}{*}{$d$} & \multirow{2}{*}{$d^{-}$} & \multicolumn{3}{|c|}{$\mathrm{ILS}_{\mathrm{RCC}}$} & \multicolumn{3}{|c|}{ ILS $_{\text {adapt }}$} & \multirow{2}{*}{$t_{\text {avg }}$} \\
\hline & & & $\min$ & avg & $\max$ & $\min$ & avg & $\max$ & \\
\hline 100 & 0.1 & 0.1 & 0.82 & 5.02 & 11.00 & 3.23 & 17.65 & 34.73 & 8.99 \\
\hline 100 & 0.1 & 0.3 & 0.02 & 1.57 & 2.49 & 1.18 & 8.17 & 16.24 & 12.60 \\
\hline 100 & 0.1 & 0.5 & 0.02 & 2.27 & 4.59 & 0.76 & 6.29 & 10.99 & 13.25 \\
\hline 100 & 0.2 & 0.1 & 0.69 & 1.35 & 1.66 & 1.62 & 4.92 & 7.46 & 10.57 \\
\hline 100 & 0.2 & 0.3 & 0.00 & 0.71 & 1.36 & 0.10 & 2.78 & 4.56 & 15.64 \\
\hline 100 & 0.2 & 0.5 & 0.11 & 1.14 & 2.67 & 0.62 & 3.08 & 5.73 & 15.19 \\
\hline 100 & 0.5 & 0.1 & 0.12 & 0.35 & 0.69 & 0.35 & 1.33 & 2.53 & 12.23 \\
\hline 100 & 0.5 & 0.3 & 0.00 & 0.21 & 0.53 & 0.00 & 0.93 & 1.98 & 21.70 \\
\hline 100 & 0.5 & 0.5 & 0.15 & 0.43 & 0.74 & 0.27 & 1.27 & 2.17 & 20.98 \\
\hline 100 & 0.8 & 0.1 & 0.00 & 0.11 & 0.28 & 0.22 & 0.95 & 1.88 & 8.91 \\
\hline
\end{tabular}




\begin{tabular}{|c|c|c|c|c|c|c|c|c|c|}
\hline \multirow{2}{*}{$|V|$} & \multirow{2}{*}{$d$} & \multirow{2}{*}{$d^{-}$} & \multicolumn{3}{|c|}{ ILS $_{\mathrm{RCC}}$} & \multicolumn{3}{|c|}{ ILS $_{\text {adapt }}$} & \multirow{2}{*}{$\mathrm{t}_{\mathrm{avg}}$} \\
\hline & & & $\min$ & avg & $\max$ & $\min$ & avg & $\max$ & \\
\hline 100 & 0.8 & 0.3 & 0.05 & 0.22 & 0.37 & 0.03 & 0.53 & 1.08 & 23.18 \\
\hline 100 & 0.8 & 0.5 & 0.00 & 0.21 & 0.34 & 0.29 & 1.10 & 1.81 & 24.85 \\
\hline 200 & 0.1 & 0.1 & 0.52 & 0.87 & 1.13 & 1.23 & 6.24 & 13.75 & 66.34 \\
\hline 200 & 0.1 & 0.3 & 0.03 & 0.81 & 1.29 & 0.94 & 2.75 & 3.65 & 97.29 \\
\hline 200 & 0.1 & 0.5 & 0.39 & 0.60 & 0.88 & 1.03 & 3.13 & 5.10 & 91.26 \\
\hline 200 & 0.2 & 0.1 & 0.15 & 0.73 & 1.40 & 0.37 & 2.34 & 4.23 & 73.44 \\
\hline 200 & 0.2 & 0.3 & 0.16 & 0.28 & 0.41 & 0.18 & 1.35 & 2.12 & 116.49 \\
\hline 200 & 0.2 & 0.5 & 0.19 & 0.44 & 0.67 & 0.73 & 1.47 & 2.01 & 102.96 \\
\hline 200 & 0.5 & 0.1 & 0.04 & 0.20 & 0.32 & 0.22 & 0.78 & 1.11 & 66.67 \\
\hline 200 & 0.5 & 0.3 & 0.05 & 0.13 & 0.21 & 0.07 & 0.52 & 0.81 & 155.52 \\
\hline 200 & 0.5 & 0.5 & 0.05 & 0.21 & 0.33 & 0.34 & 0.75 & 1.05 & 142.09 \\
\hline 200 & 0.8 & 0.1 & 0.01 & 0.11 & 0.19 & 0.12 & 0.52 & 0.84 & 44.26 \\
\hline 200 & 0.8 & 0.3 & 0.01 & 0.15 & 0.35 & 0.14 & 0.50 & 0.83 & 161.70 \\
\hline 200 & 0.8 & 0.5 & 0.04 & 0.19 & 0.28 & 0.52 & 0.85 & 1.47 & 165.27 \\
\hline 400 & 0.1 & 0.1 & 0.34 & 0.56 & 0.73 & 0.34 & 1.01 & 1.49 & 508.86 \\
\hline 400 & 0.1 & 0.3 & 0.16 & 0.31 & 0.57 & 0.09 & 0.84 & 1.45 & 870.00 \\
\hline 400 & 0.1 & 0.5 & 0.09 & 0.42 & 0.71 & 0.32 & 1.26 & 2.56 & 699.45 \\
\hline 400 & 0.2 & 0.1 & 0.07 & 0.17 & 0.20 & 0.12 & 0.38 & 0.65 & 394.25 \\
\hline 400 & 0.2 & 0.3 & 0.04 & 0.17 & 0.26 & 0.08 & 0.81 & 1.83 & 988.04 \\
\hline 400 & 0.2 & 0.5 & 0.11 & 0.23 & 0.32 & 0.26 & 0.59 & 0.76 & 778.40 \\
\hline 400 & 0.5 & 0.1 & 0.05 & 0.10 & 0.17 & 0.14 & 0.26 & 0.41 & 312.75 \\
\hline 400 & 0.5 & 0.3 & 0.03 & 0.06 & 0.07 & 0.06 & 0.14 & 0.24 & 978.23 \\
\hline 400 & 0.5 & 0.5 & 0.08 & 0.12 & 0.18 & 0.21 & 0.47 & 0.92 & 1093.10 \\
\hline 400 & 0.8 & 0.1 & 0.02 & 0.05 & 0.08 & 0.07 & 0.13 & 0.20 & 183.53 \\
\hline 400 & 0.8 & 0.3 & 0.02 & 0.05 & 0.07 & 0.12 & 0.15 & 0.19 & 881.24 \\
\hline 400 & 0.8 & 0.5 & 0.06 & 0.11 & 0.16 & 0.13 & 0.30 & 0.40 & 1304.41 \\
\hline 600 & 0.1 & 0.1 & 0.27 & 0.40 & 0.52 & 0.20 & 0.65 & 1.03 & 1338.98 \\
\hline 600 & 0.1 & 0.3 & 0.14 & 0.21 & 0.35 & 0.18 & 0.69 & 1.27 & 3173.07 \\
\hline 600 & 0.1 & 0.5 & 0.15 & 0.29 & 0.50 & 0.94 & 1.43 & 1.94 & 2470.35 \\
\hline 600 & 0.2 & 0.1 & 0.12 & 0.15 & 0.20 & 0.26 & 0.32 & 0.39 & 1034.79 \\
\hline 600 & 0.2 & 0.3 & 0.06 & 0.11 & 0.14 & 0.10 & 0.42 & 0.76 & 3313.68 \\
\hline 600 & 0.2 & 0.5 & 0.14 & 0.22 & 0.27 & 0.38 & 0.56 & 0.73 & 2824.27 \\
\hline 600 & 0.5 & 0.1 & 0.03 & 0.07 & 0.10 & 0.06 & 0.16 & 0.25 & 782.57 \\
\hline 600 & 0.5 & 0.3 & 0.02 & 0.05 & 0.08 & 0.05 & 0.12 & 0.17 & 2822.82 \\
\hline 600 & 0.5 & 0.5 & 0.04 & 0.06 & 0.11 & 0.21 & 0.30 & 0.36 & 3866.90 \\
\hline 600 & 0.8 & 0.1 & 0.02 & 0.04 & 0.06 & 0.03 & 0.06 & 0.10 & 474.08 \\
\hline 600 & 0.8 & 0.3 & 0.02 & 0.05 & 0.06 & 0.04 & 0.08 & 0.11 & 2170.92 \\
\hline 600 & 0.8 & 0.5 & 0.05 & 0.09 & 0.13 & 0.18 & 0.30 & 0.40 & 4681.35 \\
\hline
\end{tabular}

The results obtained show that, on average, ILSRCC clearly outperforms ILS $_{\text {adapt }}$. When evaluating the performance of each individual instance, the ILS $_{\mathrm{RCC}}$ found the best solution (one with a gap of $0 \%$ ) for 179 instances $(93.2 \%$ of the cases), where among them 166 (86.5\% of the cases) are strictly better than the best ones achieved by ILS adapt. Furthermore, we can also see that the average runtime increases with the value of $d^{-}$.

Figure 10 illustrates how the average gap between the average solution and the best known solution varies according to different values of $d, d^{-}$and $k$. We can observe that the instances appear to become easier when the value of $d$ increases, as depicted in Figure 10a. Furthermore, from Figure 10b, it is visible that the instances with a smaller value of $d^{-}$appear to be harder. Finally, larger values of $k$ seem to increase the difficulty of the instances, as clearly shown in Figure 10c.

In Appendix A, we also report many improved upper bounds w.r.t those obtained in the experiment reported in Table 11. These improved solutions were 


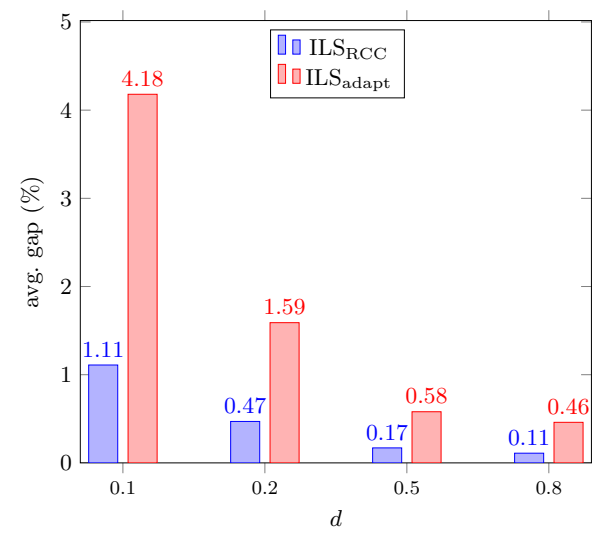

(a)

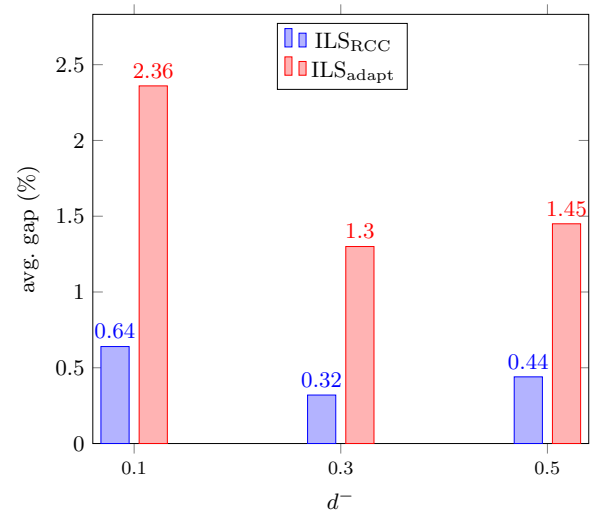

(b)

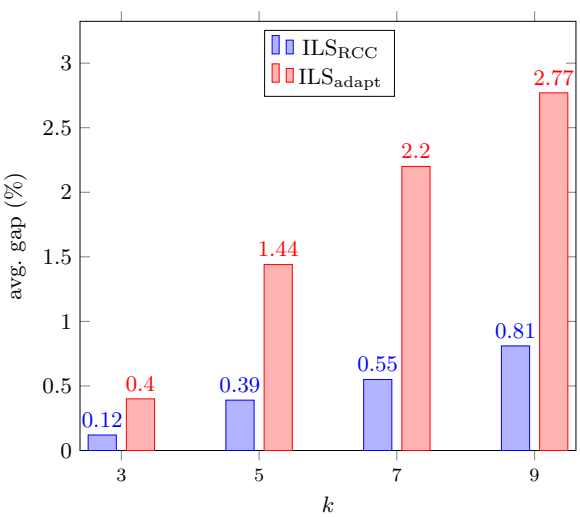

(c)

Fig. 10: Average gap performance according to characteristics of the instance.

found while experimenting with different settings of the algorithm, and also during the preliminary experiments described in Section 6.3.1.

\subsubsection{Impact of the ADSs on the runtime performance}

This section examines the average runtime performance of ILS $_{\mathrm{RCC}}$ when incorporating the ADSs for efficiently computing the relaxed imbalance value of a neighbor solution during the local search.

Figure 11 depicts the CPU time of the versions of the algorithm using EBI and NBI, respectively, in the log scale. In Figure 11a, we illustrate the comparison for the small-size Monastery instances. Despite the considerable runtime difference, it can be seen that using NBI, i.e., the one that does not make use of ADSs to perform move evaluation, is still doable in practice, as the average CPU time spent by the method is fairly acceptable. However, for the 100-vertex instances, the difference is astonishing, and visibly illustrates the benefits of incorporating 
the ADSs proposed in this work. Note that the disparity is likely to become even more prominent for larger instances.

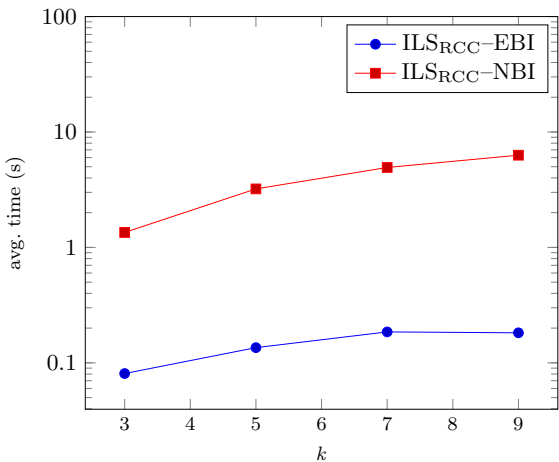

(a) Monastery instances (18 vertices)

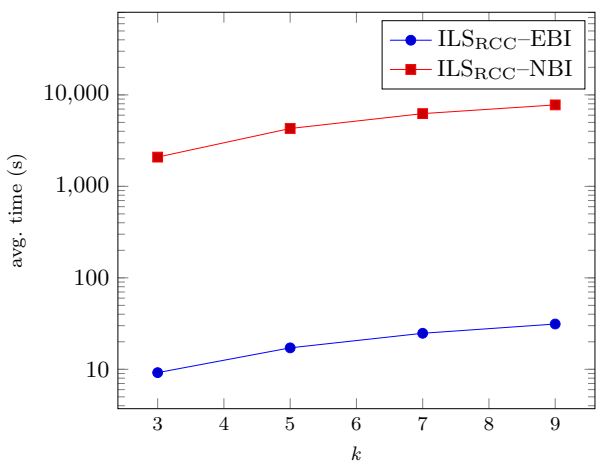

(b) Random instances with 100 vertices

Fig. 11: Impact of the ADSs on the average CPU time (semi-log plot).

\subsubsection{Results for the symmetric RCC instances}

Table 12 shows the summary of the results obtained for each set of benchmark instances. In this case, because the original algorithm from the literature is used, we refer to it as "ILS Levorato et al. [36]". Detailed results are provided in Appendix B. We report the number of strictly best solutions found by each version (\# best), the number of cases in which the best solution found by each algorithm were equal (ties), the average percentage gap (gapavg) and the minimum, average and maximum CPU time, considering the average values of 10 runs for each instance and

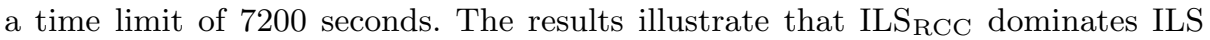
Levorato et al. [36] in terms of strictly best known solutions found, especially in Slashdot and Brazilian Congress benchmarks. To our knowledge, all best solutions found in this experiment are the best known.

Table 12: Summary of results for SRCC benchmarks

\begin{tabular}{|c|c|c|c|c|c|c|c|c|c|}
\hline \multirow{2}{*}{ Benchmark } & \multirow{2}{*}{ total } & \multicolumn{2}{|c|}{ ILS $_{\mathrm{RCC}}$} & \multicolumn{2}{|c|}{ ILS Levorato et al. [36] } & \multirow[b]{2}{*}{ ties } & \multicolumn{3}{|c|}{ time } \\
\hline & & \#best & gapavg & \#best & gapavg & & $\min$ & avg & $\max$ \\
\hline UNGA & 63 & 1 & 0.02 & 0 & 1.72 & 62 & 0.5 & 4.6 & 13.0 \\
\hline Slashdot & 7 & 7 & 9.72 & 0 & 40.08 & 0 & 16.9 & 2293.3 & 7200.0 \\
\hline BR Congress & 14 & 8 & 0.59 & 0 & 1.16 & 6 & 61.3 & 232.7 & 534.1 \\
\hline
\end{tabular}

\section{Concluding remarks}

This paper proposes exact and metaheuristic approaches for the relaxed correlation clustering (RCC) problem. In particular, we developed two integer linear 
programming formulations that obtained a superior performance when compared to the existing one, as well as an enhanced iterated local search (ILS) algorithm that substantially outperformed the previous ILS implementation from the literature. One key factor of our ILS is the efficient move evaluation scheme, which was crucial for improving the scalability of the method. Moreover, we also put forward a novel perturbation mechanism for the problem that helped the algorithm to find high quality solutions. The performance of ILS was also assessed in benchmark instances of the symmetrical version of RCC (SRCC) and the results achieved were always at least as good as the best known.

Future work includes the development of efficient parallel algorithms for tackling very large instances that may arise in real-life social networks. In addition, as the current integer linear programming formulations are still limited to small-size instances, there is still room for developing enhanced exact algorithms, perhaps in the spirit of Brusco et al. [9], as an attempt to solve larger instances.

\section{Acknowledgments}

This study was financed in part by the Coordenação de Aperfeiçoamento de Pessoal de Nível Superior - Brasil (CAPES) - Finance Code 001 and by the Conselho Nacional de Desenvolvimento Cientfífico e Tecnológico (CNPq), grants 305223/2015-1 and 303799/2018-8. We would also like to thank Mário Levorato for providing the SRCC source code and Pedro Liguori for the helpful insights on the mathematical models.

\section{Data availability}

The datasets analysed during the current study are available from the corresponding author on reasonable request.

\section{References}

1. Ales Z, Knippel A, Pauchet A (2016) Polyhedral combinatorics of the $k$ partitioning problem with representative variables. Discret Appl Math 211:114

2. Altafini C (2012) Dynamics of opinion forming in structurally balanced social networks. PLOS ONE 7(6):1-9

3. Arinik N, Figueiredo R, Labatut V (2017) Signed Graph Analysis for the Interpretation of Voting Behavior. In: International Conference on Knowledge Technologies and Data-driven Business (i-KNOW), Graz, Austria, International Workshop on Social Network Analysis and Digital Humanities (SnanDig)

4. Bahiense L, Frota Y, Maculan N, Noronha TF, Ribeiro CC (2009) A branchand-cut algorithm for equitable coloring based on a formulation by representatives. Electron Notes in Discret Math 35:347-352

5. Bansal N, Blum A, Chawla S (2004) Correlation clustering. Mach Learn 56(1):89-113 
6. Beier T, Hamprecht FA, Kappes JH (2015) Fusion moves for correlation clustering. In: CVPR. Proceedings, pp 3507-3516, 1

7. Bonami P, Nguyen VH, Klein M, Minoux M (2012) On the solution of a graph partitioning problem under capacity constraints. In: Mahjoub AR, Markakis V, Milis I, Paschos VT (eds) Combinatorial Optimization, Springer Berlin Heidelberg, Berlin, Heidelberg, pp 285-296

8. Broder AZ (1984) The r-stirling numbers. Discrete Mathematics 49(3):241 259

9. Brusco M, Doreian P, Mrvar A, Steinley D (2011) Two algorithms for relaxed structural balance partitioning: Linking theory, models, and data to understand social network phenomena. Sociol Methods \& Res 40(1):57-87

10. Brusco MJ, Doreian P (2019) Partitioning signed networks using relocation heuristics, tabu search, and variable neighborhood search. Soc Netw 56:70 80

11. Bulhões T, de Sousa Filho GF, Subramanian A, Lucídio dos Anjos FC (2017) Branch-and-cut approaches for p-cluster editing. Discret Appl Math 219:51-64

12. Campêlo M, Campos VA, Correa RC (2008) On the asymmetric representatives formulation for the vertex coloring problem. Discret Appl Math 156:10971111

13. Campêlo MB, Corrêa RC, Frota Y (2004) Cliques, holes and the vertex coloring polytope. Inf Process Lett 89(4):159-164

14. Cartwright D, Harary F (1956) Structural balance: a generalization of heider's theory. Psychological review 63(5):277

15. Dambacher JM, Li HW, Rossignol PA (2002) Relevance of community structure in assessing indeterminacy of ecological predictions. Ecol 83(5):1372-1385

16. DasGupta B, AEnciso G, Sontag E, Zhang Y (2007) Algorithmic and complexity results for decompositions of biological networks into monotone subsystems. BioSyst 90:161-178

17. Davis JA (1967) Clustering and structural balance in graphs. Human relations 20(2):181-187

18. Doreian P (2008) A multiple indicator approach to blockmodeling signed networks. Soc Netw 30(3):247 - 258

19. Doreian P, Mrvar A (1996) A partitioning approach to structural balance. Soc Netw 18(2):149 - 168

20. Doreian P, Mrvar A (2009) Partitioning signed social networks. Soc Netw 31(1):1- 11

21. Doreian P, Mrvar A (2014) Testing two theories for generating signed networks using real data

22. Doreian P, Mrvar A (2015) Structural balance and signed international relations. J of Soc Struct 16:2

23. Facchetti G, Iacono G, Altafini C (2011) Computing global structural balance in large-scale signed social networks. Proc of the National Acad of Sci 108(52):20953-20958

24. Fan N, Pardalos PM (2010) Linear and quadratic programming approaches for the general graph partitioning problem. J of Glob Optim 48(1):57-71

25. Figueiredo R, Frota Y (2014) The maximum balanced subgraph of a signed graph: Applications and solution approaches. Eur J of Oper Res 236(2):473 487 
26. Figueiredo R, Moura G (2013) Mixed integer programming formulations for clustering problems related to structural balance. Soc Netw 35(4):639 - 651

27. Figueiredo R, Frota Y, Labb M (2018) A branch-and-cut algorithm for the maximum k-balanced subgraph of a signed graph. Discret Appl Math

28. Frota Y, Maculan N, Noronha TF, Ribeiro CC (2010) A branch-and-cut algorithm for partition coloring. Netw 55:194-204

29. Harary F, Lim M, Wunsch DC (2003) Signed graphs for portfolio analysis in risk management. IMA J of Manag Math 13:1-10

30. Heider F (1946) Attitudes and cognitive organization. The Journal of Psychology 21(1):107-112, pMID: 21010780

31. Kim S, Nowozin S, Kohli P, Yoo CD (2011) Higher-order correlation clustering for image segmentation. In: Shawe-Taylor J, Zemel RS, Bartlett PL, Pereira F, Weinberger KQ (eds) Advances in Neural Information Processing Systems 24, Curran Associates, Inc., pp 1530-1538

32. Lemann TB, Solomon RL (1952) Group characteristics as revealed in sociometric patterns and personality ratings. Sociom 15(1/2):7-90

33. Levorato M (2015) Efficient solutions to the correlation clustering problem. Master's thesis, Universidade Federal Fluminense, Niterói, Rio de Janeiro, Brazil, available at http://www.ic.uff.br/PosGraduacao/ frontend-tesesdissertacoes/download.php?id=700.pdf\&tipo=trabalho

34. Levorato M, Frota Y (2017) Brazilian congress structural balance analysis. J of Interdiscip Methodol and Issues in Sci

35. Levorato M, Drummond L, Frota Y, Figueiredo R (2015) An ils algorithm to evaluate structural balance in signed social networks. In: Proceedings of the 30th Annual ACM Symposium on Applied Computing, ACM, New York, NY, USA, SAC '15, pp 1117-1122

36. Levorato M, Figueiredo R, Frota Y, Drummond L (2017) Evaluating balancing on social networks through the efficient solution of correlation clustering problems. EURO J on Comput Optim 5(4):467-498

37. Lourenço HR, Martin OC, Stützle T (2010) Iterated local search: Framework and applications. In: Gendreau M, Potvin JY (eds) Handbook of Metaheuristics, Springer US, Boston, MA, pp 363-397

38. Maurya MR, Rengaswamy R, Venkatasubramanian V (2004) Application of signed digraphs-based analysis for fault diagnosis of chemical process flowsheets. Eng Appl of Artif Intell 17(5):501 - 518

39. McKinney JC (1948) An educational application of a two-dimensional sociometric test. Sociom 11(4):356-367

40. Mladenović N, Hansen P (1997) Variable neighborhood search. Comput \& Oper Res 24(11):1097-1100

41. Newcomb TM (1961) The acquaintance process. Holt, Rinehart \& Winston, New York

42. Sampson SF (1968) A novitiate in a period of change: An experimental and case study of social relationships. PhD thesis, Department of Sociology, Cornell University, NY

43. Silva MM, Subramanian A, Vidal T, Ochi LS (2012) A simple and effective metaheuristic for the minimum latency problem. Eur J of Oper Res 221(3):513 $-520$

44. Silva MM, Subramanian A, Ochi LS (2015) An iterated local search heuristic for the split delivery vehicle routing problem. Comput \& Oper Res 53:234 - 
249

45. Subramanian A, Farias K (2017) Efficient local search limitation strategy for single machine total weighted tardiness scheduling with sequence-dependent setup times. Comput \& Oper Res 79:190 - 206

46. Van Gael J, Zhu X (2007) Correlation clustering for crosslingual link detection. In: Proceedings of the 20th International Joint Conference on Artifical Intelligence, Morgan Kaufmann Publishers Inc., San Francisco, CA, USA, IJCAI'07, pp 1744-1749

47. Vasanthi B, Arumugam S, Nagar AK, Mitra S (2015) Applications of signed graphs to portfolio turnover analysis. Procedia - Soc and Behav Sci 211:1203 1209, 2nd Global Conference on Business and Social Sciences (GCBSS-2015) on Multidisciplinary Perspectives on Management and Society, 17- 18 September, 2015, Bali, Indonesia

48. Wang N, Li J (2013) Restoring: A greedy heuristic approach based on neighborhood for correlation clustering. In: Motoda H, Wu Z, Cao L, Zaiane O, Yao M, Wang W (eds) Advanced Data Mining and Applications, Springer Berlin Heidelberg, Berlin, Heidelberg, pp 348-359

49. Yang B, Cheung W, Liu J (2007) Community mining from signed social networks. IEEE Trans on Knowl and Data Eng 19:1333-1348

50. Zaslavsky T (1982) Signed graphs. Discret Appl Math 4:47-74

51. Zaslavsky T (1998) A mathematical bibliography of signed and gain graphs and allied areas. Electron $\mathrm{J}$ of Comb DS8 
A Detailed results for the random RCC instances

Table 13: Relaxed imbalance obtained by ILS $_{\mathrm{RCC}}$ and ILS $_{\text {adapt }}$.

\begin{tabular}{|c|c|c|c|c|c|c|c|c|c|c|}
\hline \multirow{2}{*}{$|V|$} & \multirow{2}{*}{$d$} & \multirow{2}{*}{$d^{-}$} & \multirow{2}{*}{$k$} & \multicolumn{3}{|c|}{$\mathrm{ILS}_{\mathrm{RCC}}$} & \multicolumn{3}{|c|}{ ILS $_{\text {adapt }}$} & \multirow[b]{2}{*}{$\mathrm{t}_{\mathrm{avg}}$} \\
\hline & & & & $\min$ & avg & $\max$ & $\min$ & avg & $\max$ & \\
\hline 100 & 0.1 & 0.1 & 3 & 131 & 132.10 & 134 & 133 & 135.40 & 139 & 2.82 \\
\hline 100 & 0.1 & 0.1 & 5 & 94 & 98.20 & 101 & 103 & 108.50 & 112 & 6.71 \\
\hline 100 & 0.1 & 0.1 & 7 & 68 & 70.90 & 74 & 78 & 84.40 & 89 & 11.80 \\
\hline 100 & 0.1 & 0.1 & 9 & 45 & 50.70 & 54 & 66 & 69.00 & 73 & 14.65 \\
\hline 100 & 0.1 & 0.3 & 3 & 408 & 408.10 & 409 & 409 & 412.90 & 416 & 5.73 \\
\hline 100 & 0.1 & 0.3 & 5 & 323 & 327.50 & 333 & 335 & 342.60 & 348 & 11.05 \\
\hline 100 & 0.1 & 0.3 & 7 & 270 & 276.70 & 284 & 280 & 294.00 & 305 & 15.84 \\
\hline 100 & 0.1 & 0.3 & 9 & 233 & 239.00 & 247 & 252 & 257.60 & 263 & 17.78 \\
\hline 100 & 0.1 & 0.5 & 3 & 533 & 533.10 & 534 & 533 & 537.10 & 546 & 6.93 \\
\hline 100 & 0.1 & 0.5 & 5 & 427 & 430.70 & 435 & 435 & 446.60 & 457 & 11.98 \\
\hline 100 & 0.1 & 0.5 & 7 & 352 & 365.30 & 371 & 380 & 387.10 & 397 & 15.10 \\
\hline 100 & 0.1 & 0.5 & 9 & 298 & 312.50 & 324 & 325 & 334.90 & 341 & 18.99 \\
\hline 100 & 0.2 & 0.1 & 3 & 327 & 329.30 & 333 & 327 & 332.40 & 336 & 2.83 \\
\hline 100 & 0.2 & 0.1 & 5 & 284 & 288.80 & 291 & 293 & 297.00 & 302 & 7.22 \\
\hline 100 & 0.2 & 0.1 & 7 & 253 & 256.70 & 261 & 263 & 269.90 & 275 & 13.10 \\
\hline 100 & 0.2 & 0.1 & 9 & 227 & 230.70 & 233 & 234 & 245.40 & 252 & 19.12 \\
\hline 100 & 0.2 & 0.3 & 3 & 1032 & 1032.00 & 1032 & 1032 & 1033.00 & 1035 & 5.88 \\
\hline 100 & 0.2 & 0.3 & 5 & 913 & 914.90 & 917 & 927 & 937.10 & 948 & 14.35 \\
\hline 100 & 0.2 & 0.3 & 7 & 833 & 843.70 & 854 & 847 & 866.80 & 876 & 19.09 \\
\hline 100 & 0.2 & 0.3 & 9 & 775 & 785.70 & 799 & 797 & 812.10 & 821 & 23.22 \\
\hline 100 & 0.2 & 0.5 & 3 & 1341 & 1342.50 & 1344 & 1346 & 1349.40 & 1358 & 7.87 \\
\hline 100 & 0.2 & 0.5 & 5 & 1162 & 1171.40 & 1177 & 1182 & 1194.90 & 1204 & 13.43 \\
\hline 100 & 0.2 & 0.5 & 7 & 1057 & 1067.60 & 1075 & 1070 & 1092.30 & 1105 & 17.56 \\
\hline 100 & 0.2 & 0.5 & 9 & 955 & 981.30 & 998 & 994 & 1013.10 & 1023 & 21.90 \\
\hline 100 & 0.5 & 0.1 & 3 & 940 & 941.10 & 943 & 940 & 943.30 & 947 & 3.81 \\
\hline 100 & 0.5 & 0.1 & 5 & 899 & 901.00 & 903 & 902 & 906.50 & 910 & 8.74 \\
\hline 100 & 0.5 & 0.1 & 7 & 864 & 867.30 & 873 & 871 & 878.20 & 883 & 14.85 \\
\hline 100 & 0.5 & 0.1 & 9 & 833 & 838.80 & 843 & 844 & 854.70 & 871 & 21.54 \\
\hline 100 & 0.5 & 0.3 & 3 & 2741 & 2741.00 & 2741 & 2741 & 2741.10 & 2742 & 7.57 \\
\hline 100 & 0.5 & 0.3 & 5 & 2629 & 2631.20 & 2634 & 2632 & 2636.70 & 2642 & 19.69 \\
\hline 100 & 0.5 & 0.3 & 7 & 2532 & 2537.90 & 2556 & 2555 & 2568.80 & 2581 & 28.54 \\
\hline 100 & 0.5 & 0.3 & 9 & 2459 & 2472.10 & 2487 & 2497 & 2508.60 & 2524 & 31.01 \\
\hline 100 & 0.5 & 0.5 & 3 & 3939 & 3944.90 & 3958 & 3939 & 3949.80 & 3966 & 12.35 \\
\hline 100 & 0.5 & 0.5 & 5 & 3659 & 3677.70 & 3693 & 3697 & 3713.10 & 3729 & 19.50 \\
\hline 100 & 0.5 & 0.5 & 7 & 3507 & 3518.90 & 3529 & 3537 & 3548.70 & 3559 & 25.56 \\
\hline 100 & 0.5 & 0.5 & 9 & 3343 & 3367.90 & 3382 & 3399 & 3417.10 & 3435 & 26.51 \\
\hline 100 & 0.8 & 0.1 & 3 & 1537 & 1538.00 & 1541 & 1537 & 1540.40 & 1546 & 2.82 \\
\hline 100 & 0.8 & 0.1 & 5 & 1502 & 1502.00 & 1502 & 1503 & 1509.50 & 1515 & 6.08 \\
\hline 100 & 0.8 & 0.1 & 7 & 1470 & 1471.30 & 1474 & 1473 & 1484.00 & 1493 & 10.41 \\
\hline 100 & 0.8 & 0.1 & 9 & 1439 & 1443.10 & 1445 & 1448 & 1456.40 & 1465 & 16.35 \\
\hline 100 & 0.8 & 0.3 & 3 & 4599 & 4601.50 & 4606 & 4599 & 4600.60 & 4604 & 7.17 \\
\hline 100 & 0.8 & 0.3 & 5 & 4462 & 4468.90 & 4476 & 4468 & 4478.60 & 4488 & 18.14 \\
\hline 100 & 0.8 & 0.3 & 7 & 4364 & 4377.30 & 4385 & 4369 & 4391.50 & 4403 & 30.49 \\
\hline 100 & 0.8 & 0.3 & 9 & 4270 & 4286.10 & 4308 & 4303 & 4316.60 & 4332 & 36.91 \\
\hline 100 & 0.8 & 0.5 & 3 & 6676 & 6676.00 & 6676 & 6676 & 6695.60 & 6741 & 16.14 \\
\hline 100 & 0.8 & 0.5 & 5 & 6319 & 6330.80 & 6347 & 6343 & 6376.80 & 6401 & 23.40 \\
\hline 100 & 0.8 & 0.5 & 7 & 6095 & 6115.60 & 6141 & 6136 & 6166.90 & 6195 & 28.12 \\
\hline 100 & 0.8 & 0.5 & 9 & 5921 & 5939.60 & 5959 & 5973 & 5989.00 & 6008 & 31.76 \\
\hline 200 & 0.1 & 0.1 & 3 & 691 & 694.60 & 700 & 695 & 699.60 & 703 & 16.03 \\
\hline 200 & 0.1 & 0.1 & 5 & 602 & 606.70 & 611 & 615 & 624.30 & 634 & 42.44 \\
\hline 200 & 0.1 & 0.1 & 7 & 539 & 545.20 & 550 & 565 & 576.10 & 582 & 85.12 \\
\hline 200 & 0.1 & 0.1 & 9 & 499 & 504.30 & 511 & 524 & 535.90 & 544 & 121.75 \\
\hline 200 & 0.1 & 0.3 & 3 & 1993 & 1993.50 & 1995 & 2002 & 2011.90 & 2024 & 32.91 \\
\hline 200 & 0.1 & 0.3 & 5 & 1782 & 1799.30 & 1812 & 1823 & 1834.90 & 1848 & 90.46 \\
\hline 200 & 0.1 & 0.3 & 7 & 1659 & 1680.70 & 1695 & 1712 & 1719.70 & 1730 & 123.25 \\
\hline 200 & 0.1 & 0.3 & 9 & 1574 & 1589.70 & 1607 & 1619 & 1633.60 & 1645 & 142.54 \\
\hline 200 & 0.1 & 0.5 & 3 & 2716 & 2726.60 & 2744 & 2732 & 2744.20 & 2754 & 56.06 \\
\hline 200 & 0.1 & 0.5 & 5 & 2403 & 2414.90 & 2435 & 2435 & 2465.90 & 2484 & 81.47 \\
\hline 200 & 0.1 & 0.5 & 7 & 2226 & 2240.50 & 2260 & 2274 & 2299.50 & 2327 & 108.14 \\
\hline 200 & 0.1 & 0.5 & 9 & 2104 & 2122.70 & 2135 & 2151 & 2173.70 & 2189 & 119.36 \\
\hline 200 & 0.2 & 0.1 & 3 & 1515 & 1517.30 & 1526 & 1516 & 1520.70 & 1524 & 15.82 \\
\hline 200 & 0.2 & 0.1 & 5 & 1422 & 1433.80 & 1445 & 1437 & 1452.50 & 1461 & 43.79 \\
\hline
\end{tabular}




\begin{tabular}{|c|c|c|c|c|c|c|c|c|c|c|}
\hline \multirow{2}{*}{$|V|$} & \multirow{2}{*}{$d$} & \multirow{2}{*}{$d^{-}$} & \multirow{2}{*}{$k$} & \multicolumn{3}{|c|}{ ILS $_{\mathrm{RCC}}$} & \multicolumn{3}{|c|}{ ILS ${ }_{\text {adapt }}$} & \multirow{2}{*}{$\mathrm{t}_{\mathrm{avg}}$} \\
\hline & & & & $\min$ & avg & $\max$ & $\min$ & avg & $\max$ & \\
\hline 200 & 0.2 & 0.1 & 7 & 1357 & 1364.40 & 1371 & 1384 & 1394.30 & 1410 & 88.66 \\
\hline 200 & 0.2 & 0.1 & 9 & 1293 & 1311.50 & 1326 & 1328 & 1350.10 & 1357 & 145.51 \\
\hline 200 & 0.2 & 0.3 & 3 & 4376 & 4383.10 & 4387 & 4377 & 4384.10 & 4392 & 32.41 \\
\hline 200 & 0.2 & 0.3 & 5 & 4136 & 4144.30 & 4154 & 4161 & 4176.80 & 4194 & 102.09 \\
\hline 200 & 0.2 & 0.3 & 7 & 3957 & 3971.30 & 3988 & 4027 & 4042.70 & 4054 & 163.24 \\
\hline 200 & 0.2 & 0.3 & 9 & 3856 & 3871.90 & 3885 & 3916 & 3938.90 & 3960 & 168.21 \\
\hline 200 & 0.2 & 0.5 & 3 & 6142 & 6153.80 & 6178 & 6172 & 6187.30 & 6206 & 66.70 \\
\hline 200 & 0.2 & 0.5 & 5 & 5689 & 5715.00 & 5729 & 5740 & 5772.30 & 5818 & 92.31 \\
\hline 200 & 0.2 & 0.5 & 7 & 5436 & 5460.90 & 5501 & 5449 & 5528.80 & 5558 & 114.75 \\
\hline 200 & 0.2 & 0.5 & 9 & 5229 & 5264.30 & 5283 & 5298 & 5336.40 & 5362 & 138.09 \\
\hline 200 & 0.5 & 0.1 & 3 & 3950 & 3951.50 & 3957 & 3952 & 3958.90 & 3963 & 20.22 \\
\hline 200 & 0.5 & 0.1 & 5 & 3876 & 3887.10 & 3899 & 3893 & 3903.90 & 3912 & 43.74 \\
\hline 200 & 0.5 & 0.1 & 7 & 3814 & 3826.40 & 3835 & 3835 & 3855.60 & 3869 & 77.27 \\
\hline 200 & 0.5 & 0.1 & 9 & 3769 & 3774.70 & 3782 & 3802 & 3811.20 & 3824 & 125.47 \\
\hline 200 & 0.5 & 0.3 & 3 & 11624 & 11629.30 & 11639 & 11624 & 11632.30 & 11643 & 43.78 \\
\hline 200 & 0.5 & 0.3 & 5 & 11368 & 11384.70 & 11398 & 11398 & 11416.20 & 11436 & 112.73 \\
\hline 200 & 0.5 & 0.3 & 7 & 11169 & 11193.00 & 11217 & 11227 & 11254.90 & 11273 & 213.97 \\
\hline 200 & 0.5 & 0.3 & 9 & 11052 & 11066.70 & 11089 & 11122 & 11142.10 & 11175 & 251.60 \\
\hline 200 & 0.5 & 0.5 & 3 & 17090 & 17098.40 & 17124 & 17121 & 17147.80 & 17192 & 104.95 \\
\hline 200 & 0.5 & 0.5 & 5 & 16367 & 16401.30 & 16462 & 16434 & 16496.40 & 16540 & 136.04 \\
\hline 200 & 0.5 & 0.5 & 7 & 15969 & 16006.10 & 16038 & 16054 & 16099.80 & 16137 & 157.54 \\
\hline 200 & 0.5 & 0.5 & 9 & 15621 & 15673.30 & 15710 & 15734 & 15787.00 & 15821 & 169.82 \\
\hline 200 & 0.8 & 0.1 & 3 & 6348 & 6348.80 & 6350 & 6348 & 6355.50 & 6366 & 14.59 \\
\hline 200 & 0.8 & 0.1 & 5 & 6284 & 6291.10 & 6295 & 6297 & 6310.60 & 6322 & 29.45 \\
\hline 200 & 0.8 & 0.1 & 7 & 6226 & 6237.90 & 6247 & 6243 & 6263.90 & 6279 & 52.70 \\
\hline 200 & 0.8 & 0.1 & 9 & 6181 & 6189.70 & 6202 & 6199 & 6214.10 & 6234 & 80.28 \\
\hline 200 & 0.8 & 0.3 & 3 & 18810 & 18811.80 & 18816 & 18817 & 18823.60 & 18831 & 41.21 \\
\hline 200 & 0.8 & 0.3 & 5 & 18546 & 18556.70 & 18569 & 18573 & 18588.90 & 18612 & 116.73 \\
\hline 200 & 0.8 & 0.3 & 7 & 18339 & 18374.70 & 18400 & 18381 & 18415.50 & 18450 & 197.81 \\
\hline 200 & 0.8 & 0.3 & 9 & 18148 & 18211.30 & 18241 & 18261 & 18291.50 & 18319 & 291.05 \\
\hline 200 & 0.8 & 0.5 & 3 & 28291 & 28301.70 & 28311 & 28305 & 28364.70 & 28410 & 129.39 \\
\hline 200 & 0.8 & 0.5 & 5 & 27342 & 27400.10 & 27458 & 27497 & 27544.10 & 27589 & 157.90 \\
\hline 200 & 0.8 & 0.5 & 7 & 26793 & 26867.10 & 26911 & 26921 & 27000.30 & 27078 & 180.17 \\
\hline 200 & 0.8 & 0.5 & 9 & 26416 & 26474.00 & 26534 & 26521 & 26591.40 & 26676 & 193.61 \\
\hline 400 & 0.1 & 0.1 & 3 & 2989 & 2996.30 & 3006 & 2986 & 2996.10 & 3003 & 104.42 \\
\hline 400 & 0.1 & 0.1 & 5 & 2812 & 2826.60 & 2839 & 2813 & 2833.70 & 2853 & 284.54 \\
\hline 400 & 0.1 & 0.1 & 7 & 2669 & 2688.60 & 2711 & 2687 & 2708.50 & 2756 & 625.85 \\
\hline 400 & 0.1 & 0.1 & 9 & 2569 & 2586.10 & 2605 & 2578 & 2608.00 & 2633 & 1020.60 \\
\hline 400 & 0.1 & 0.3 & 3 & 8843 & 8851.50 & 8862 & 8837 & 8845.30 & 8854 & 233.79 \\
\hline 400 & 0.1 & 0.3 & 5 & 8360 & 8374.40 & 8383 & 8389 & 8413.10 & 8475 & 811.53 \\
\hline 400 & 0.1 & 0.3 & 7 & 8022 & 8047.40 & 8074 & 8040 & 8119.80 & 8206 & 1280.75 \\
\hline 400 & 0.1 & 0.3 & 9 & 7835 & 7880.20 & 7934 & 7893 & 7950.30 & 7990 & 1153.92 \\
\hline 400 & 0.1 & 0.5 & 3 & 12523 & 12532.80 & 12544 & 12522 & 12562.00 & 12610 & 508.94 \\
\hline 400 & 0.1 & 0.5 & 5 & 11611 & 11648.60 & 11679 & 11622 & 11734.00 & 11783 & 660.74 \\
\hline 400 & 0.1 & 0.5 & 7 & 11099 & 11178.80 & 11209 & 11179 & 11243.30 & 11309 & 815.20 \\
\hline 400 & 0.1 & 0.5 & 9 & 10807 & 10868.90 & 10928 & 10869 & 10929.00 & 10985 & 812.93 \\
\hline 400 & 0.2 & 0.1 & 3 & 6296 & 6300.10 & 6306 & 6296 & 6303.30 & 6315 & 91.57 \\
\hline 400 & 0.2 & 0.1 & 5 & 6147 & 6159.20 & 6170 & 6157 & 6167.90 & 6186 & 247.20 \\
\hline 400 & 0.2 & 0.1 & 7 & 6028 & 6039.90 & 6071 & 6035 & 6054.00 & 6085 & 462.77 \\
\hline 400 & 0.2 & 0.1 & 9 & 5912 & 5923.90 & 5934 & 5927 & 5950.60 & 5975 & 775.45 \\
\hline 400 & 0.2 & 0.3 & 3 & 18278 & 18284.70 & 18298 & 18277 & 18291.60 & 18305 & 235.19 \\
\hline 400 & 0.2 & 0.3 & 5 & 17735 & 17758.10 & 17779 & 17764 & 17802.00 & 17849 & 786.29 \\
\hline 400 & 0.2 & 0.3 & 7 & 17385 & 17431.00 & 17467 & 17509 & 17555.30 & 17597 & 1370.41 \\
\hline 400 & 0.2 & 0.3 & 9 & 17163 & 17207.10 & 17236 & 17244 & 17321.80 & 17373 & 1560.29 \\
\hline 400 & 0.2 & 0.5 & 3 & 26898 & 26921.80 & 26941 & 26892 & 26960.90 & 27041 & 567.47 \\
\hline 400 & 0.2 & 0.5 & 5 & 25577 & 25655.80 & 25711 & 25707 & 25774.00 & 25805 & 749.25 \\
\hline 400 & 0.2 & 0.5 & 7 & 24947 & 24994.40 & 25055 & 25016 & 25108.90 & 25199 & 846.28 \\
\hline 400 & 0.2 & 0.5 & 9 & 24472 & 24550.60 & 24590 & 24530 & 24639.40 & 24727 & 950.62 \\
\hline 400 & 0.5 & 0.1 & 3 & 15853 & 15860.50 & 15867 & 15865 & 15875.60 & 15885 & 100.59 \\
\hline 400 & 0.5 & 0.1 & 5 & 15758 & 15767.10 & 15777 & 15775 & 15786.70 & 15811 & 208.04 \\
\hline 400 & 0.5 & 0.1 & 7 & 15642 & 15668.50 & 15684 & 15665 & 15689.90 & 15717 & 361.93 \\
\hline 400 & 0.5 & 0.1 & 9 & 15561 & 15580.80 & 15610 & 15602 & 15625.00 & 15641 & 580.47 \\
\hline 400 & 0.5 & 0.3 & 3 & 47486 & 47500.60 & 47521 & 47488 & 47516.40 & 47536 & 228.52 \\
\hline 400 & 0.5 & 0.3 & 5 & 47004 & 47020.20 & 47038 & 46996 & 47035.40 & 47074 & 666.00 \\
\hline 400 & 0.5 & 0.3 & 7 & 46604 & 46636.50 & 46677 & 46632 & 46682.40 & 46759 & 1295.69 \\
\hline 400 & 0.5 & 0.3 & 9 & 46319 & 46352.50 & 46381 & 46353 & 46429.70 & 46474 & 1722.73 \\
\hline 400 & 0.5 & 0.5 & 3 & 71795 & 71852.80 & 71898 & 71858 & 71969.60 & 72029 & 955.35 \\
\hline
\end{tabular}




\begin{tabular}{|c|c|c|c|c|c|c|c|c|c|c|}
\hline \multirow{2}{*}{$|V|$} & \multirow{2}{*}{$d$} & \multirow{2}{*}{$d^{-}$} & \multirow{2}{*}{$k$} & \multicolumn{3}{|c|}{ ILS $_{\mathrm{RCC}}$} & \multicolumn{3}{|c|}{ ILS $_{\text {adapt }}$} & \multirow{2}{*}{$\mathrm{t}_{\mathrm{avg}}$} \\
\hline & & & & $\min$ & avg & $\max$ & $\min$ & avg & $\max$ & \\
\hline 400 & 0.5 & 0.5 & 5 & 69825 & 69888.40 & 69958 & 69973 & 70148.90 & 70274 & 1105.49 \\
\hline 400 & 0.5 & 0.5 & 7 & 68747 & 68873.20 & 68939 & 68945 & 69082.80 & 69205 & 1116.31 \\
\hline 400 & 0.5 & 0.5 & 9 & 68093 & 68167.30 & 68262 & 68103 & 68239.80 & 68522 & 1195.26 \\
\hline 400 & 0.8 & 0.1 & 3 & 25285 & 25289.20 & 25293 & 25295 & 25302.50 & 25310 & 68.99 \\
\hline 400 & 0.8 & 0.1 & 5 & 25192 & 25203.60 & 25210 & 25205 & 25220.40 & 25242 & 134.38 \\
\hline 400 & 0.8 & 0.1 & 7 & 25086 & 25106.80 & 25124 & 25095 & 25135.10 & 25170 & 209.22 \\
\hline 400 & 0.8 & 0.1 & 9 & 25027 & 25038.30 & 25051 & 25054 & 25064.40 & 25081 & 321.54 \\
\hline 400 & 0.8 & 0.3 & 3 & 76029 & 76044.80 & 76075 & 76039 & 76084.70 & 76157 & 214.40 \\
\hline 400 & 0.8 & 0.3 & 5 & 75601 & 75627.60 & 75674 & 75605 & 75673.40 & 75748 & 547.72 \\
\hline 400 & 0.8 & 0.3 & 7 & 75228 & 75280.40 & 75332 & 75237 & 75315.60 & 75396 & 1099.86 \\
\hline 400 & 0.8 & 0.3 & 9 & 74910 & 74954.10 & 74982 & 74960 & 75032.90 & 75096 & 1662.96 \\
\hline 400 & 0.8 & 0.5 & 3 & 117476 & 117543.50 & 117626 & 117527 & 117631.00 & 117773 & 1109.08 \\
\hline 400 & 0.8 & 0.5 & 5 & 114831 & 114990.90 & 115126 & 115077 & 115261.10 & 115489 & 1294.47 \\
\hline 400 & 0.8 & 0.5 & 7 & 113596 & 113681.80 & 113790 & 113660 & 113938.50 & 114126 & 1407.46 \\
\hline 400 & 0.8 & 0.5 & 9 & 112546 & 112725.80 & 112904 & 112836 & 112996.20 & 113288 & 1406.61 \\
\hline 600 & 0.1 & 0.1 & 3 & 6842 & 6854.10 & 6861 & 6833 & 6847.00 & 6858 & 317.03 \\
\hline 600 & 0.1 & 0.1 & 5 & 6621 & 6632.10 & 6643 & 6614 & 6642.60 & 6678 & 736.46 \\
\hline 600 & 0.1 & 0.1 & 7 & 6405 & 6438.80 & 6474 & 6438 & 6464.60 & 6501 & 1656.70 \\
\hline 600 & 0.1 & 0.1 & 9 & 6251 & 6283.30 & 6298 & 6291 & 6316.40 & 6359 & 2645.73 \\
\hline 600 & 0.1 & 0.3 & 3 & 20349 & 20382.00 & 20398 & 20356 & 20385.00 & 20412 & 783.90 \\
\hline 600 & 0.1 & 0.3 & 5 & 19655 & 19682.20 & 19706 & 19712 & 19755.70 & 19788 & 2518.19 \\
\hline 600 & 0.1 & 0.3 & 7 & 19168 & 19201.50 & 19228 & 19238 & 19324.00 & 19372 & 4519.86 \\
\hline 600 & 0.1 & 0.3 & 9 & 18775 & 18840.30 & 18875 & 18913 & 19016.70 & 19078 & 4870.33 \\
\hline 600 & 0.1 & 0.5 & 3 & 29305 & 29349.80 & 29379 & 29379 & 29451.00 & 29516 & 2034.70 \\
\hline 600 & 0.1 & 0.5 & 5 & 27696 & 27765.20 & 27866 & 27923 & 27959.40 & 28017 & 2364.64 \\
\hline 600 & 0.1 & 0.5 & 7 & 26880 & 26956.00 & 27029 & 27007 & 27129.90 & 27196 & 2621.14 \\
\hline 600 & 0.1 & 0.5 & 9 & 26244 & 26374.90 & 26442 & 26352 & 26501.40 & 26600 & 2860.93 \\
\hline 600 & 0.2 & 0.1 & 3 & 14166 & 14171.30 & 14180 & 14154 & 14172.20 & 14178 & 262.04 \\
\hline 600 & 0.2 & 0.1 & 5 & 13976 & 13996.50 & 14030 & 13969 & 14007.40 & 14053 & 626.76 \\
\hline 600 & 0.2 & 0.1 & 7 & 13812 & 13830.10 & 13862 & 13830 & 13866.80 & 13894 & 1196.27 \\
\hline 600 & 0.2 & 0.1 & 9 & 13662 & 13683.90 & 13707 & 13674 & 13711.30 & 13758 & 2054.11 \\
\hline 600 & 0.2 & 0.3 & 3 & 42106 & 42129.40 & 42160 & 42115 & 42149.60 & 42189 & 751.15 \\
\hline 600 & 0.2 & 0.3 & 5 & 41340 & 41395.50 & 41442 & 41425 & 41464.50 & 41531 & 2294.29 \\
\hline 600 & 0.2 & 0.3 & 7 & 40801 & 40856.90 & 40912 & 40951 & 41016.00 & 41092 & 4377.53 \\
\hline 600 & 0.2 & 0.3 & 9 & 40406 & 40450.90 & 40490 & 40584 & 40716.60 & 40825 & 5831.76 \\
\hline 600 & 0.2 & 0.5 & 3 & 62367 & 62455.70 & 62532 & 62487 & 62603.90 & 62704 & 2301.21 \\
\hline 600 & 0.2 & 0.5 & 5 & 60041 & 3.50 & 60337 & 16 & 604 & 60597 & 2740.62 \\
\hline 600 & 0.2 & 0.5 & 7 & 58939 & 59089.90 & 59212 & 59165 & 59296.90 & 59363 & 3095.99 \\
\hline 600 & 0.2 & 0.5 & 9 & 58124 & 58245.80 & 58399 & 58270 & 58433.80 & 58576 & 3159.25 \\
\hline 600 & 0.5 & 0.1 & 3 & 35888 & 35897.40 & 35910 & 35893 & 35911.10 & 35920 & 265.25 \\
\hline 600 & 0.5 & 0.1 & 5 & 35748 & 35764.60 & 35779 & 35784 & 35799.60 & 35814 & 574.68 \\
\hline 600 & 0.5 & 0.1 & 7 & 35614 & 35649.50 & 35677 & 35659 & 35678.20 & 35701 & 874.83 \\
\hline 600 & 0.5 & 0.1 & 9 & 35493 & 35527.30 & 35551 & 35543 & 35580.60 & 35608 & 1415.54 \\
\hline 600 & 0.5 & 0.3 & 3 & 106944 & 106962.10 & 106982 & 106940 & 106989.50 & 107042 & 664.15 \\
\hline 600 & 0.5 & 0.3 & 5 & 106282 & 106328.70 & 106368 & 106297 & 106375.70 & 106493 & 1943.99 \\
\hline 600 & 0.5 & 0.3 & 7 & 105701 & 105777.80 & 105879 & 105767 & 105879.30 & 106002 & 3598.85 \\
\hline 600 & 0.5 & 0.3 & 9 & 105256 & 105341.80 & 105417 & 105298 & 105424.20 & 105562 & 5084.31 \\
\hline 600 & 0.5 & 0.5 & 3 & 165124 & 165197.50 & 165260 & 165337 & 165464.60 & 165612 & 3580.68 \\
\hline 600 & 0.5 & 0.5 & 5 & 161478 & 161564.70 & 161669 & 161907 & 162056.50 & 162160 & 3874.33 \\
\hline 600 & 0.5 & 0.5 & 7 & 159537 & 159707.10 & 159876 & 159824 & 160116.70 & 160347 & 3929.42 \\
\hline 600 & 0.5 & 0.5 & 9 & 158301 & 158384.50 & 158506 & 158513 & 158763.00 & 159063 & 4083.18 \\
\hline 600 & 0.8 & 0.1 & 3 & 57334 & 57346.90 & 57357 & 57333 & 57352.90 & 57369 & 166.32 \\
\hline 600 & 0.8 & 0.1 & 5 & 57208 & 57225.60 & 57248 & 57231 & 57242.50 & 57251 & 352.95 \\
\hline 600 & 0.8 & 0.1 & 7 & 57044 & 57075.50 & 57106 & 57044 & 57099.50 & 57179 & 524.58 \\
\hline 600 & 0.8 & 0.1 & 9 & 56968 & 56990.30 & 57006 & 56971 & 57003.20 & 57061 & 852.47 \\
\hline 600 & 0.8 & 0.3 & 3 & 172024 & 172049.90 & 172084 & 172064 & 172094.00 & 172142 & 575.18 \\
\hline 600 & 0.8 & 0.3 & 5 & 171515 & 171559.10 & 171605 & 171459 & 171590.80 & 171695 & 1375.56 \\
\hline 600 & 0.8 & 0.3 & 7 & 170996 & 171088.70 & 171165 & 171044 & 171165.60 & 171259 & 2608.47 \\
\hline 600 & 0.8 & 0.3 & 9 & 170559 & 170648.70 & 170738 & 170651 & 170742.30 & 170905 & 4124.47 \\
\hline 600 & 0.8 & 0.5 & 3 & 269158 & 269304.00 & 269465 & 269353 & 269646.40 & 269850 & 4247.83 \\
\hline 600 & 0.8 & 0.5 & 5 & 264210 & 264560.20 & 264822 & 265003 & 265276.20 & 265560 & 4722.79 \\
\hline 600 & 0.8 & 0.5 & 7 & 261978 & 262158.00 & 262388 & 262582 & 262903.30 & 263149 & 4998.81 \\
\hline 600 & 0.8 & 0.5 & 9 & 260310 & 260584.20 & 260937 & 260654 & 260983.80 & 261283 & 4755.96 \\
\hline
\end{tabular}


Table 14: Best solution of all experiments.

\begin{tabular}{|c|c|c|c|c|}
\hline$|V|$ & $d$ & $d^{-}$ & $k$ & $R I(P)$ \\
\hline 100 & 0.1 & 0.1 & 7 & 64 \\
\hline 100 & 0.1 & 0.1 & 9 & 44 \\
\hline 100 & 0.1 & 0.3 & 5 & 319 \\
\hline 100 & 0.1 & 0.3 & 7 & 265 \\
\hline 100 & 0.1 & 0.3 & 9 & 220 \\
\hline 100 & 0.1 & 0.5 & 5 & 421 \\
\hline 100 & 0.1 & 0.5 & 7 & 350 \\
\hline 100 & 0.1 & 0.5 & 9 & 297 \\
\hline 100 & 0.2 & 0.1 & 7 & 250 \\
\hline 100 & 0.2 & 0.1 & 9 & 223 \\
\hline 100 & 0.2 & 0.3 & 7 & 832 \\
\hline 100 & 0.2 & 0.3 & 9 & 772 \\
\hline 100 & 0.2 & 0.5 & 7 & 1047 \\
\hline 100 & 0.5 & 0.1 & 7 & 863 \\
\hline 100 & 0.5 & 0.3 & 7 & 2526 \\
\hline 100 & 0.5 & 0.3 & 9 & 2455 \\
\hline 100 & 0.5 & 0.5 & 7 & 3486 \\
\hline 100 & 0.5 & 0.5 & 9 & 3326 \\
\hline 100 & 0.8 & 0.1 & 7 & 1468 \\
\hline 100 & 0.8 & 0.3 & 7 & 4356 \\
\hline 100 & 0.8 & 0.5 & 7 & 6089 \\
\hline 100 & 0.8 & 0.5 & 9 & 5882 \\
\hline 200 & 0.1 & 0.1 & 3 & 689 \\
\hline 200 & 0.1 & 0.1 & 9 & 492 \\
\hline 200 & 0.1 & 0.3 & 7 & 1656 \\
\hline 200 & 0.1 & 0.5 & 5 & 2397 \\
\hline 200 & 0.1 & 0.5 & 9 & 2092 \\
\hline 200 & 0.2 & 0.1 & 7 & 1354 \\
\hline 200 & 0.2 & 0.3 & 5 & 4133 \\
\hline 200 & 0.2 & 0.5 & 5 & 5686 \\
\hline 200 & 0.2 & 0.5 & 9 & 5212 \\
\hline 200 & 0.5 & 0.3 & 9 & 11012 \\
\hline 200 & 0.5 & 0.5 & 7 & 15944 \\
\hline 200 & 0.8 & 0.5 & 5 & 27326 \\
\hline 400 & 0.1 & 0.1 & 7 & 2666 \\
\hline 400 & 0.1 & 0.1 & 9 & 2566 \\
\hline 400 & 0.1 & 0.3 & 5 & 8356 \\
\hline 400 & 0.1 & 0.3 & 7 & 8003 \\
\hline 400 & 0.1 & 0.5 & 3 & 12506 \\
\hline 400 & 0.1 & 0.5 & 5 & 11597 \\
\hline 400 & 0.2 & 0.1 & 7 & 6014 \\
\hline 400 & 0.2 & 0.1 & 9 & 5900 \\
\hline 400 & 0.2 & 0.3 & 3 & 18265 \\
\hline 400 & 0.2 & 0.3 & 5 & 17715 \\
\hline 400 & 0.2 & 0.3 & 9 & 17127 \\
\hline 400 & 0.2 & 0.5 & 3 & 26860 \\
\hline 400 & 0.2 & 0.5 & 5 & 25572 \\
\hline 400 & 0.2 & 0.5 & 7 & 24909 \\
\hline 400 & 0.2 & 0.5 & 9 & 24460 \\
\hline 400 & 0.5 & 0.1 & 5 & 15756 \\
\hline 400 & 0.5 & 0.3 & 3 & 47475 \\
\hline 400 & 0.5 & 0.3 & 9 & 46291 \\
\hline 400 & 0.5 & 0.5 & 5 & 69762 \\
\hline 400 & 0.5 & 0.5 & 9 & 68037 \\
\hline 400 & 0.8 & 0.1 & 5 & 25186 \\
\hline 400 & 0.8 & 0.3 & 7 & 75216 \\
\hline 400 & 0.8 & 0.3 & 9 & 74907 \\
\hline
\end{tabular}




\begin{tabular}{ccccr}
\multicolumn{5}{c}{ Table $14-$ Continued from } \\
\hline$|V|$ & $d$ & $d^{-}$ & $k$ & $R I(P)$ \\
\hline 600 & 0.1 & 0.1 & 5 & 6612 \\
600 & 0.1 & 0.3 & 5 & 19649 \\
600 & 0.1 & 0.3 & 7 & 19145 \\
600 & 0.1 & 0.5 & 3 & 29275 \\
600 & 0.2 & 0.1 & 7 & 13810 \\
600 & 0.2 & 0.1 & 9 & 13652 \\
600 & 0.2 & 0.3 & 3 & 42100 \\
600 & 0.2 & 0.3 & 5 & 41333 \\
600 & 0.2 & 0.3 & 7 & 40755 \\
600 & 0.2 & 0.3 & 9 & 40391 \\
600 & 0.2 & 0.5 & 3 & 62337 \\
600 & 0.2 & 0.5 & 9 & 58122 \\
600 & 0.5 & 0.1 & 3 & 35886 \\
600 & 0.5 & 0.3 & 3 & 106938 \\
600 & 0.5 & 0.3 & 5 & 106272 \\
600 & 0.5 & 0.3 & 9 & 105211 \\
600 & 0.5 & 0.5 & 3 & 165046 \\
600 & 0.5 & 0.5 & 5 & 161377 \\
600 & 0.5 & 0.5 & 9 & 158300 \\
600 & 0.8 & 0.1 & 9 & 56940 \\
600 & 0.8 & 0.3 & 7 & 170971
\end{tabular}




\section{B Detailed results for the SRCC instances}

Table 15: Symmetric relaxed imbalance obtained by ILS $_{\mathrm{RCC}}$ and ILS Levorato et al. [36].

\begin{tabular}{|c|c|c|c|c|c|c|c|c|c|c|c|}
\hline \multirow{2}{*}{ Instance } & \multirow{2}{*}{$|V|$} & \multirow{2}{*}{$d$} & \multirow{2}{*}{$d^{-}$} & \multirow{2}{*}{$k$} & \multicolumn{3}{|c|}{$\mathrm{ILS}_{\mathrm{RCC}}$} & \multicolumn{3}{|c|}{ ILS Levorato et al. [36] } & \multirow{2}{*}{$\mathrm{t}_{\mathrm{avg}}$} \\
\hline & & & & & $\min$ & avg & $\max$ & $\min$ & avg & $\max$ & \\
\hline UNGA-1946 & 54 & 0.484 & 0.27 & 2 & 9.338 & 9.338 & 9.338 & 9.338 & 9.338 & 9.338 & 0.5 \\
\hline UNGA-1947 & 57 & 0.490 & 0.42 & 3 & 18.698 & 18.697 & 18.698 & 18.698 & 18.697 & 18.698 & 0.7 \\
\hline UNGA-1948 & 59 & 0.494 & 0.34 & 4 & 4.399 & 4.399 & 4.399 & 4.399 & 4.399 & 4.399 & 1.2 \\
\hline UNGA-1949 & 59 & 0.496 & 0.28 & 2 & 37.748 & 37.748 & 37.748 & 37.748 & 37.748 & 37.748 & 0.6 \\
\hline UNGA-1950 & 60 & 0.496 & 0.25 & 2 & 25.028 & 25.028 & 25.028 & 25.028 & 25.028 & 25.028 & 0.6 \\
\hline UNGA-1951 & 60 & 0.490 & 0.37 & 2 & 58.960 & 58.960 & 58.960 & 58.960 & 58.960 & 58.960 & 0.8 \\
\hline $\mathrm{UNC}$ & 60 & 0.495 & 0.26 & 2 & 99 & 46. & 46.099 & 46.099 & 46.099 & 46.099 & 0.6 \\
\hline UNGA-1953 & 60 & 0.488 & 0.34 & 2 & 31.288 & 31.288 & 31.288 & 31.288 & 31.288 & 31.288 & 0.8 \\
\hline UNGA-1954 & 60 & 0.492 & 0.30 & 2 & 32.823 & 32.823 & 32.823 & 32.823 & 32.823 & 32.823 & 0.8 \\
\hline UNGA-1955 & 65 & 0.464 & 0.11 & 4 & 5.377 & 5.377 & 5.377 & 5.377 & 5.377 & 5.377 & 1.5 \\
\hline UNGA-1956 & 81 & 0.480 & 0.30 & 4 & 17.181 & 17.181 & 17.181 & 17.181 & 17.181 & 17.181 & 2.3 \\
\hline UNGA-1957 & 82 & 0.495 & 0.32 & 3 & 37.512 & 37.512 & 37.512 & 37.512 & 37.512 & 37.512 & 2.0 \\
\hline UNGA-1958 & 82 & 0.489 & 0.25 & 2 & 122.536 & 122.536 & 122.536 & 122.536 & 122.536 & 122.536 & 1.2 \\
\hline UNGA-1959 & 82 & 0.497 & 0.35 & 2 & 102.881 & 102.881 & 102.881 & 102.881 & 102.881 & 102.881 & 2.2 \\
\hline UNGA-1960 & 100 & 0.488 & 0.39 & 3 & 45.464 & 45.464 & 45.464 & 45.464 & 45.464 & 45.464 & 3.4 \\
\hline UNGA-1961 & 106 & 0.467 & 0.35 & 3 & 37.395 & 37.395 & 37.395 & 37.395 & 37.395 & 37.395 & 3.9 \\
\hline UNGA & 110 & 0.468 & 0.33 & 2 & 154.412 & 154.412 & 154.412 & 154.412 & 154.412 & 154.412 & 1.4 \\
\hline UNGA-1963 & 113 & 0.490 & 0.18 & 4 & 20.639 & 20.639 & 20.639 & 20.639 & 20.639 & 20.639 & 3.7 \\
\hline UNGA- $1964^{1}$ & 115 & 0.500 & 0.28 & 3 & 000 & 0.000 & 0.000 & 0.000 & 31.200 & 39.000 & 1.0 \\
\hline UNGA-1965 & 117 & 0.495 & 0.21 & 4 & & 29 & & 29.482 & .482 & 29.482 & 6.3 \\
\hline UNG & 122 & 0.484 & 0.23 & 2 & 21 & 213. & 21 & 213.680 & 213.680 & 213.680 & 2.3 \\
\hline UN & 124 & 0.490 & 0.29 & 4 & & 8 & 98 & 42.298 & 298 & 42.298 & 7.4 \\
\hline UNG & 126 & 0.490 & 0.25 & 3 & 39 & 86. & 39 & 86.239 & 239 & 86.239 & 6.1 \\
\hline UNG & 126 & 0.495 & 0.21 & 3 & 77 & 66. & .277 & 66.277 & 66.277 & 66.277 & 4.9 \\
\hline UNGA-1970 & 127 & 0.497 & 0.21 & 3 & .316 & 69.316 & 9.316 & 69.316 & 69.316 & 69.316 & 4.9 \\
\hline UNGA-1971 & 133 & 0.493 & 0.09 & 4 & 19.306 & 19.306 & 9.306 & 19.306 & 19.306 & 19.306 & 4.8 \\
\hline UNGA-1972 & 132 & 0.499 & 0.04 & 2 & 6.294 & 16.294 & 6.294 & 16.294 & 16.294 & 16.294 & 1.4 \\
\hline UNGA-1973 & 135 & 0.499 & 0.09 & 3 & 1.142 & 14.142 & 4.142 & 14.142 & 14.142 & 14.142 & 2.5 \\
\hline UNC & 138 & 0.499 & 0.10 & 3 & 608 & 18.608 & 3.608 & 18.608 & 3.608 & 18.608 & 3.1 \\
\hline 975 & 143 & 0.472 & 0.21 & 4 & & & 07 & 53.707 & .707 & 53.707 & 6.6 \\
\hline UN & 144 & 0.460 & 0.16 & 4 & & & & 06 & 506 & 606 & 5.6 \\
\hline 77 & 146 & 0.465 & 0.09 & 6 & & 8 & 48 & 15.548 & 548 & 15.548 & 12.7 \\
\hline UN & 148 & 0.483 & 0.14 & 3 & 7 & 5 & 55 & 75.445 & 629 & 77.812 & 3.1 \\
\hline $\mathrm{UN}$ & 150 & 0.470 & 0.16 & 5 & & & 20 & 21.520 & 520 & 21.520 & 9.6 \\
\hline UNGA & 151 & 0.474 & 0.18 & 6 & 03 & 03 & 03 & 29.303 & 30.054 & 31.807 & 11.7 \\
\hline UNGA-1981 & 155 & 0.477 & 0.18 & 5 & 9.121 & 29.121 & 9.121 & 29.121 & 29.121 & 29.121 & 11.3 \\
\hline UNGA-1982 & 156 & 0.432 & 0.15 & 4 & 1.378 & 31.378 & 1.378 & 31.378 & 31.378 & 31.378 & 9.8 \\
\hline UNGA-1983 & 157 & 0.474 & 0.22 & 4 & .523 & 29.523 & .523 & 29.523 & 36.168 & 62.750 & 7.4 \\
\hline UNGA-1984 & 158 & 0.439 & 0.20 & 4 & .033 & 14. & 4.033 & 4.033 & 17.197 & 45.679 & 6.9 \\
\hline UNGA-1985 & 158 & 0.431 & 0.12 & 2 & 30 & 53. & 30 & 3.630 & 3.630 & 53.630 & 2.9 \\
\hline UN & 158 & 0.499 & 0.08 & 2 & & & & 44.673 & 673 & 44.673 & 2.4 \\
\hline UNGA & 158 & 0.499 & 0.05 & 3 & & & & 19 & 19 & 9.119 & 2.8 \\
\hline UN & 158 & 0.499 & 0.08 & 2 & & & & 05 & 05 & 33.905 & 2.5 \\
\hline UN & 158 & 0.500 & 0.05 & 2 & & & & 02 & & 17.302 & 2.3 \\
\hline UNG & 158 & 0.498 & 0.10 & 3 & & & 24 & 24 & 024 & 15.024 & 3.5 \\
\hline UNC & 178 & 0.467 & 0. & 3 & 80 & 0 & 80 & 15.480 & 669 & 15.692 & 3.6 \\
\hline UNGA-1992 & 180 & 0.493 & 0.08 & 4 & 2.201 & 12.201 & 12.201 & 12.201 & 12.770 & 17.889 & 8.0 \\
\hline UNGA-1993 & 184 & 0.496 & 0.09 & 3 & 4.689 & 24.972 & 25.003 & 24.689 & 24.689 & 24.689 & 4.4 \\
\hline UNGA-1994 & 185 & 0.497 & 0.1 & 3 & 3.573 & 23.573 & 23.573 & 23.573 & 23.573 & 23.573 & 5.5 \\
\hline UNGA-1995 & 185 & 0.489 & 0.11 & 3 & 27.624 & 27.624 & 27.624 & 27.624 & 28.248 & 28.663 & 5.1 \\
\hline UNGA-1996 & 185 & 0.499 & 0.07 & 3 & 9.541 & 9.541 & 9.541 & 9.541 & 9.541 & 9.541 & 5.6 \\
\hline UNGA-1997 & 176 & 0.471 & 0.16 & 5 & .018 & 7.018 & 7.018 & 27.018 & 7.018 & 27.018 & 13.0 \\
\hline UNGA-1998 & 177 & 0.492 & 0.14 & 4 & 39.300 & 39.300 & 39.300 & 39.300 & 39.300 & 39.300 & 11.2 \\
\hline UNGA-1999 & 182 & 0.487 & 0.10 & 4 & 14.375 & 14.375 & 14.375 & 14.375 & 14.386 & 14.412 & 9.0 \\
\hline UNG & 189 & 0.495 & 0.13 & 4 & 25.099 & 25.100 & 25.099 & 25.099 & 25.100 & 25.099 & 8.6 \\
\hline UNC & 191 & 0.495 & 0.16 & 2 & 33.531 & 33.531 & 33.531 & 33.531 & 33.531 & 33.531 & 6.8 \\
\hline UNGA-2002 & 192 & 0.495 & 0.10 & 3 & 12.687 & 12.687 & 12.687 & 12.687 & 12.687 & 12.687 & 4.1 \\
\hline
\end{tabular}

1 In the 19th session, voting occurred on only one resolution which explains the signed digraph with very low relaxed imbalance. 


\begin{tabular}{|c|c|c|c|c|c|c|c|c|c|c|c|}
\hline \multirow{2}{*}{ Instance } & \multirow{2}{*}{$|V|$} & \multirow{2}{*}{$d$} & \multirow{2}{*}{$d^{-}$} & \multirow{2}{*}{$k$} & \multicolumn{3}{|c|}{ ILS $_{\mathrm{RCC}}$} & \multicolumn{3}{|c|}{ ILS Levorato et al. [36] } & \multirow{2}{*}{$\mathrm{t}_{\text {avg }}$} \\
\hline & & & & & $\min$ & avg & $\overline{\max }$ & $\min$ & avg & $\overline{\max }$ & \\
\hline UNGA-2003 & 191 & 0.489 & 0.06 & 2 & 7.466 & 7.466 & 7.466 & 7.466 & 7.466 & 7.466 & 4.2 \\
\hline UNGA-2004 & 191 & 0.498 & 0.05 & 2 & 20.638 & 20.638 & 20.638 & 20.638 & 20.638 & 20.638 & 3.6 \\
\hline UNGA-2005 & 192 & 0.482 & 0.06 & 3 & 25.516 & 25.516 & 25.516 & 25.516 & 25.516 & 25.516 & 6.0 \\
\hline UNGA-2006 & 192 & 0.498 & 0.05 & 2 & 28.954 & 28.955 & 28.954 & 28.954 & 28.955 & 28.954 & 3.6 \\
\hline UNGA-2007 & 192 & 0.498 & 0.06 & 2 & 45.570 & 45.570 & 45.570 & 45.570 & 45.570 & 45.570 & 3.7 \\
\hline UNGA-2008 & 192 & 0.495 & 0.06 & 2 & 36.889 & 36.889 & 36.889 & 36.889 & 36.889 & 36.889 & 3.8 \\
\hline Slashdot1 & 200 & 0.022 & 0.07 & 5 & 11.0 & 11.700 & 12.0 & 16.0 & 17.30 & 19.0 & 16.9 \\
\hline Slashdot2 & 300 & 0.012 & 0.08 & 8 & 4.0 & 4.600 & 5.0 & 8.0 & 11.10 & 12.0 & 61.1 \\
\hline Slashdot3 & 400 & 0.008 & 0.07 & 4 & 15.0 & 16.100 & 18.0 & 20.0 & 22.20 & 24.0 & 67.7 \\
\hline Slashdot4 & 600 & 0.005 & 0.08 & 9 & 8.0 & 10.700 & 13.0 & 16.0 & 19.20 & 21.0 & 438.5 \\
\hline Slashdot5 & 800 & 0.005 & 0.11 & 20 & 14.0 & 16.300 & 20.0 & 32.0 & 35.60 & 41.0 & 2161.5 \\
\hline Slashdot6 & 1000 & 0.006 & 0.14 & 11 & 182.0 & 187.200 & 194.0 & 206.0 & 211.10 & 218.0 & 6107.6 \\
\hline Slashdot7 & 2000 & 0.005 & 0.15 & 43 & 626.0 & 650.700 & 686.0 & 712.0 & 751.90 & 777.0 & 7200.0 \\
\hline BR-2010-v1 & 545 & 0.490 & 0.01 & 4 & 309.692 & 309.692 & 09.692 & 16.091 & 375.860 & 596.137 & 35.3 \\
\hline BR-2010-v2 & 545 & 0.490 & 0.02 & 4 & 332.493 & 332. & 33 & 338.836 & 849 & 345.102 & 160.9 \\
\hline BR-2011-v1 & 553 & 0.488 & 0.20 & 4 & 562.540 & 562.541 & 562.541 & 562.540 & 584.250 & 589.677 & 534.2 \\
\hline BR-2011-v2 & 553 & 0.486 & 0.21 & 4 & 566.950 & 566.950 & 566.950 & 566.950 & 594.462 & 601.993 & 517.5 \\
\hline BR-2012-v1 & 555 & 0.489 & 0.04 & 4 & 658.613 & 658.613 & 658.613 & 672.986 & 755.450 & 1072.030 & 202.8 \\
\hline BR-2012-v2 & 555 & 0.488 & 0.04 & 4 & 681.168 & 681.168 & 681.168 & 697.567 & 703.277 & 704.705 & 202.2 \\
\hline BR-2013-v1 & 540 & 0.489 & 0.04 & 4 & 483.511 & 503.611 & 514.011 & 483.511 & 757.230 & 1266.900 & 117.1 \\
\hline BR-2013-v2 & 540 & 0.489 & 0.04 & 4 & 504.918 & 522.200 & 539.912 & 631.341 & 692.390 & 732.912 & 115.6 \\
\hline BR-2014-v1 & 556 & 0.496 & 0.01 & 4 & 130.020 & 131.001 & 131.968 & 131.973 & 136.706 & 168.493 & 61.3 \\
\hline BR-2014-v2 & 556 & 0.495 & 0.01 & 4 & 137.165 & 137.629 & 138.326 & 140.349 & 148.078 & 182.796 & 65.4 \\
\hline BR-2015-v1 & 552 & 0.486 & 0.12 & 4 & 536.724 & 536.724 & 536.724 & 536.724 & 676.785 & 1147.860 & 271.7 \\
\hline BR-2015-v2 & 552 & 0.484 & 0.18 & 4 & 585.038 & 585.038 & 585.038 & 585.038 & 707.996 & 830.955 & 294.7 \\
\hline BR-2016-v1 & 544 & 0.484 & 0.10 & 4 & 1241.520 & 1241.520 & 1241.520 & 1241.520 & 1411.645 & 1610.620 & 291.9 \\
\hline BR-2016-v2 & 544 & 0.482 & 0.11 & 4 & 1285.950 & 1285.950 & 1285.950 & 1377.020 & 1511.614 & 1677.130 & 287.7 \\
\hline
\end{tabular}

